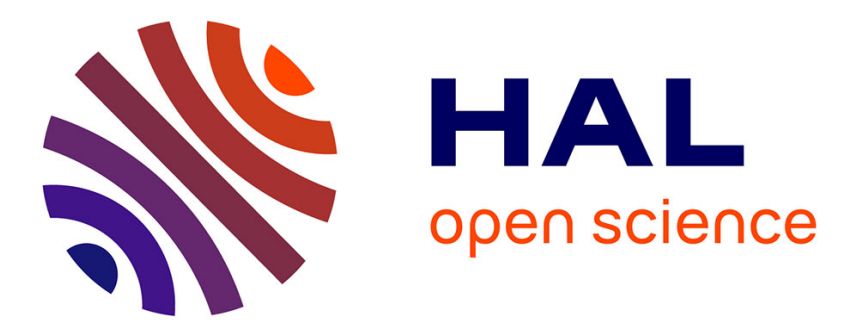

\title{
Surrogates based multi-criteria predesign methodology of Sodium-cooled Fast Reactor cores - Application to CFV-like cores
}

Olivier Fabbris, Saied Dardour, Patrick Blaise, Jean-Henry Ferrasse, Manuel Saez

\section{To cite this version:}

Olivier Fabbris, Saied Dardour, Patrick Blaise, Jean-Henry Ferrasse, Manuel Saez. Surrogates based multi-criteria predesign methodology of Sodium-cooled Fast Reactor cores - Application to CFV-like cores. Nuclear Engineering and Design, 2016, 305, pp.314-333. 10.1016/j.nucengdes.2016.05.021 . hal-01461795

\section{HAL Id: hal-01461795 \\ https://hal.science/hal-01461795}

Submitted on 15 Jul 2021

HAL is a multi-disciplinary open access archive for the deposit and dissemination of scientific research documents, whether they are published or not. The documents may come from teaching and research institutions in France or abroad, or from public or private research centers.
L'archive ouverte pluridisciplinaire $\mathbf{H A L}$, est destinée au dépôt et à la diffusion de documents scientifiques de niveau recherche, publiés ou non, émanant des établissements d'enseignement et de recherche français ou étrangers, des laboratoires publics ou privés. 


\title{
Surrogates based multi-criteria predesign methodology of Sodium-cooled Fast Reactor cores - Application to CFV-like cores
}

\author{
Olivier Fabbris ${ }^{\mathrm{a}}$, Saied Dardour ${ }^{\mathrm{a}, *}$, Patrick Blaise ${ }^{\mathrm{b}}$, Jean-Henry Ferrasse ${ }^{\mathrm{c}}$, Manuel Saez ${ }^{\mathrm{a}}$ \\ ${ }^{\text {a } C E A ~ D E N / D E R / S E S I, ~} 13108$ Saint-Paul-Lez-Durance, France \\ ${ }^{\mathrm{b}}$ CEA DEN/DER/SPEX, 13108 Saint-Paul-Lez-Durance, France \\ ${ }^{\mathrm{c}}$ Aix-Marseille Université, CNRS, ECM, M2P2 UMR 7340, 13451 Marseille, France
}

\begin{abstract}
The Sodium-cooled Fast Reactor (SFR) core predesign process is commonly realized on the basis of expert advices and local parametric studies. As such, in-deep knowledge of physical phenomena avoids an important number of expensive simulations. However, the study space is explored only partially. To ease the computational burden metamodels, or surrogate models, can be used, to quickly evaluate the performances of a wide set of different cores, individually defined by a set of parameters (pellet diameter, fissile height...), in the study space. This paper presents the development of a simplified neutronics ERANOS reference core calculation scheme that is then implemented in the construction of the Design of Experiment (DOE) database. The surrogate models for SFR CFV-like cores performances are developed, biases and uncertainties are quantified against the CFV-v1 version. Global Sensitivity Analysis also allowed highlighting antagonist performances for the design and to propose two alternative core configurations. A broadened application of the method with an optimization of a CFV-like core is also detailed. The Pareto front of the seven selected performance parameters has been studied using eleven surrogate models, based on Artificial Neural Network (ANN). The optimization demonstrates that the CFV-v1, designed using Best Estimate codes, under given performance constraints, is Pareto optimal: no other configuration is highlighted from the Multi-Objective Optimization (MOO) study. Further MOO analysis, including a specific study on impact of new degrees of freedom, such as five Pu enrichments compared to two, or different pellet diameters have been performed. Additional configurations are then found by the surrogate models, improving simultaneously all performances of the CFV-v1 configuration.
\end{abstract}

\section{Introduction}

In France, Fast Reactor strategy was confirmed in May 2008 at Ministry level and in September 2010 an agreement was published between CEA and French Government in order to conduct design studies of Generation IV (GEN IV) Sodium-cooled Fast Reactors (SFRs). An innovative core design, the CFV (French acronym for

\footnotetext{
* Corresponding author. Tel.: +334422536 77 .

E-mail address: saied.dardour@cea.fr (S. Dardour).
}

Low sodium Void effect), was first suggested by the CEA (Sciora et al., 2011) with the objective of reducing the probability of core meltdown and/or limiting energy release accident potentialities. Characterized by axial and radial heterogeneous geometry design, this particular core features a negative sodium void worth and allows improving the natural behavior during unprotected thermal hydraulics transients, such as the ULOSSP transient (Unprotected Loss of Station Supply Power). However, with low primary mechanical pump inertia (around $10 \mathrm{~s}$ for the halving time), sodium boiling might be hard to rule out if uncertainties are 
considered and propagated through the whole calculation process. For that reason, further improvement of the core behavior is under progress and should lead to a new core release.

To deal with uncertainties, safety analyses can use conservative or best-estimate codes. Conservative codes include assumptions to cover unknown uncertainties. Best Estimate (BE) codes model all the relevant processes, but are extremely time consuming.

One of the multi-scale/multi-physics international programs was the BEMUSE (Best Estimate Methods in Uncertainty and Sensitivity Evaluation) Program, promoted by the Working Group on Accident Management and Analysis (WGAMA) and endorsed by the Committee on the Safety of Nuclear Installations (CSNI). It consisted in five phases, covering LOFT (Loss of Flow Transient) and LOCA (Loss of Coolant Accident) accidents, and their associated uncertainty analysis. An overview of the project can be found in (Perez et al., 2010).

Within the frame of BEMUSE phase IV, IRSN used the CATHARE code as to build surrogate models for the analysis of Peak Cladding Temperature (PCT), one of the criteria of the LB-LOCA (Fouet et al., 2007). The approach is based on response surface methodology, constructed by Kriging.

Surrogate models are being used in nuclear engineering for more than 10 years, mainly in the field of thermal-hydraulics, where highly nonlinear phenomena require time consuming CPU resources. Hence, surrogate models are an alternative solution to the reference models as they enable, with limited computation effort, to extract quantitative information from the simulation.

The nuclear system characterization is performed by chaining/coupling several physics. Core designs results from parametric studies (Chenaud et al., 2013). Even if High Performance Computing allows today calculations with BE codes, complete and precise calculations require repeated use of computationally expensive simulations (within several days or weeks).

Recently, various attempts were made to develop more generalized optimization methods (Barjot et al., 2014). These methods allow to evaluate the behavior of a large number of cores and to select a design complying with neutron physics, thermalhydraulics, thermo-mechanics, and transient behavior requirements. However, simplifications are usually needed during the process and precision is thus reduced.

In this paper, development of surrogate models (so-called metamodels) to predesign core characteristics is detailed. In the second section, the method is reported: the general layout is first explained and the overall scheme of the method is presented. Then, options and calculation schemes used within neutron physics code are presented. Finally, the choice of the Design of Experiments (DOE) and of the regression method, called meta-model, is developed. The Section 3 develops the state of the art in SFR core predesign and optimization studies, and gives an overview of the process. The Section 4 details the development of an optimized deterministic calculation scheme and its validation. The Section 5 details the development process and the validation of the surrogate models. The built metamodels allow the detailed and exhaustive Global Sensitivity Analysis of Section 6. Section 7 presents the Multi-Objective Optimization process: the perimeter, as well as the ranking and first results is detailed. A methodology for identifying new optimized configurations is described, and the validation of a representative set from the Pareto front is also performed. In Section 8, an additional optimization study is presented, using augmented degrees of freedom.

\section{Basics of surrogates models use as a basis for Global Sensitivity Analysis (GSA)}

For complex design issues, surrogate models can be regarded as approximation models for the cost function and state function, which are built from sampled data obtained by randomly probing the design space (called sampling via DOE). Once the surrogate models are built, algorithms such as Genetic Algorithms (GA) can be used to search alternative designs (based on the surrogate models). Since the prediction with a surrogate model is generally much more efficient than with a numerical analysis code, the computational cost associated with the search based on the surrogate models is generally negligible. Surrogate modeling is referred to as a technique that makes use of the sampled data (observed by running the computer code) to build surrogate models, which are sufficient to predict the output of an expensive computer code at untried points in the design space. Thus, how to choose sample points, how to build surrogate models, and how to evaluate the accuracy of surrogate models are key issues for surrogate modeling.

\subsection{Design of Experiments}

To build a metamodel, DOE methods are usually used to determine the locations of sample points in the so called "design space", i.e., that can spread over several dozens of dimensions. DOE is a procedure whose general goal is to maximize the amount of information gained form a limited number of sample points (Giunta et al., 2001). Currently, there are different DOE methods which can be classified into two categories: "classic" DOE methods and "modern" DOE methods. The classic DOE methods, such as fullfactorial design, Central Composite Design (CCD), Box-Behnken and D-Optimal Design (DOD), were developed for the sake of laboratory experiments, with the consideration of reducing the effect of random error. By comparison, the modern DOE methods such as Latin Hypercube Design (LHD), Orthogonal Array Design (OAD) and Uniform Design (UD) were developed for deterministic computer experiments without any random error consideration as for in classic laboratory experiment's DOE. An overview of both classic and modern DOE methods was presented by Giunta et al. (2001). A more detailed description of existing DOE methods is beyond the scope of this section.

\subsection{Surrogate models construction techniques}

Metamodeling techniques have been widely developed and used in engineering design to improve the efficiency in simulation and optimization of systems that involve computationally expensive simulation programs such as aeronautics, climatology. Many existing applications are restricted to deterministic optimization. Major publications are related to these fields of research and development (Forrester et al., 2008). A comprehensive review of metamodeling applications in mechanical and aerospace systems can be found in (Simpson et al., 1997). A comparative study of various metamodeling techniques has been provided by Jin et al. (2001) using multiple modeling criteria and multiple test problems. And a whole review of their potential use for computation intensive processes is given by Wang and Shan (2005).

Metamodels are mathematical functions. The form of the function used defines the type of metamodel. Several types exist: polynomial models, Radial Basis Functions (RBF), Artificial Neural Network (ANN), splines (MARS models) or Kriging to quote the most used ones in the literature. The choice of metamodel type is driven by several criteria: number of inputs, regularity of the response function, cost, needed accuracy.

Surrogate models built from approximate simulations are also imprecise, and have to be validated against some known solutions, obtained by reference calculations. A strategy is needed for improving the precision of surrogate models based on approximate simulations without significantly increasing computational time. Moreover, as surrogate models are validated, they enable 
uncertainty propagation, in an easier - and much faster - way, from the input parameters to the output parameters.

A resume of the state-of-the art of high performance computing and possibilities of surrogate models application to multi-physics problems can be found in Turinsky's paper (Turinsky, 2012).

The present study is based on the combined use of Latin Hypercube Sampling (LHS) for the generation of the learning database, coupled with an Artificial Neural Network (ANN) for surrogate construction. The whole process is described in Section 5.

\section{Review of fast reactors design and optimization studies}

\subsection{State-of-the-art}

Several studies already exist in the field of fast reactor design. Most classical of them aim at getting in depth understanding of some physical phenomena via parametric studies and then suggest enhanced configurations based on this refined knowledge. The METRO (Mathematical Estimation of Transients for Reactor design Orientation) methodology (Stauff et al., 2013) is focused on carbide fuel behavior and reactivity coefficients to propose safety enhanced design options such as sodium bonding. Sun's work (Sun, 2012) aims at finding the best compromise for the core design by acting on four main parameters (core size, plutonium content, moderator fraction and sodium plenum size). The study is then concluded by thermal-hydraulics transient calculations.

Another approach developed in FARM (FAst Reactor Methodology) methodology (Ingremeau, 2011) consists in considering directly multi-physics aspect (neutron physics, fuel behavior, and thermal-hydraulics) to perform Multi-Objective Optimization. The complexity of the problem lead the author to base the study on analytic physics models supported by polynomial functions to model some of the physical laws driving the system. The main goal was to identify trends for the global performances of an industrial Gas-cooled Fast Reactor.

Barjot and Hourcade works on respectively SDDS (SHADOCbased Design Development System) (Barjot et al., 2014) and TRIAD (Tools for Reactor optimization Analysis and Design) (Hourcade et al., 2013) tools respectively, aim at chaining calculation scheme to build response databases. These databases are in both cases used to construct surrogate models of system response in domains such as neutron physics, thermo-mechanics and thermo-hydraulics. In the former, the metamodels are used to sample intensively the design space and identify the best configurations regarding one safety criteria at a time. The latter use a genetic algorithm to compute a set of optimal core configurations; a graphical analysis of the extreme solutions allowed authors to specify global trends for the core performances.

\subsection{Lacks in actual approaches and proposed improvement}

Regarding the previous design studies applied to fast reactor design, several aspects have not been assessed yet in existing methodologies:

- None of the study is performed in a high dimensional design space: the maximum number of parameters never exceeds ten.

- All these studies are based on simplified physical models.

- Optimality of the suggested designs is not always insured.

- Validation of the results in the surrogate based methods is not assessed.

The method presented in this paper was based on the following characteristics:
- Parameters impacts and interactions between parameters were not assumed to be known; several tens (30) of design parameters were changed simultaneously. The main goal was to develop an exploratory tool. Specific sampling of the design space, based on modern optimized space filling design, was implemented.

- The process relied on reference deterministic code, to get results as close as possible from a standard design process. For the sake of surrogate modeling though, some simplification on the reference scheme still had to be done. A systematic validation method was applied to insure the quality of the results.

- Implementation of metamodels used a specific validation database along with a sequential construction technique.

The framework of the present work is described by the general diagram depicted on Fig. 1. The steps of this methodology consist in developing an optimization suitable calculation scheme. This scheme is then use to construct databases (DOE techniques) which lead to construction of high fidelity metamodels. These regression functions are then used to get insight of the performances behavior and to propose alternative core configurations. Multi-criteria optimization results will be discussed in another paper. Future works will also extend the methodology to a calculation chain for multi-physics studies (as depicted in Fig. 1). The calculation chain covered by the present paper is colored in red.

\section{Building the neutronics model}

The neutronics surrogate models are built from a Physical Object, here the CFV-v1 core, and its translation into a model objective and assumptions. Those cases will be used to develop the learning database (Design of Experiment).

\subsection{CFV-core as a Physical Object}

French GEN IV SFR core design is mainly guided by safety objectives.

- The first one is prevention of the core meltdown accident, at first through natural favorable behavior of the core and of the system, and with addition of passive complementary systems if natural behavior is not sufficient for some transient cases.

- The second one is the mitigation of severe accidents to guarantee that core melting accidents does not lead to significant mechanical energy release.

Core design objectives are the followings:

- Natural favorable behavior for transients of Unprotected Loss of Flow (ULOF) and Unprotected Loss of Heat Sink (ULOHS), target criteria: no sodium boiling for an ULOF.

- Sodium void effect minimized (target criteria: negative sodium void effect if the core and the plenum are voided).

- Natural favorable behavior for a complete control rod withdrawal, with no detection (target criteria: no fuel pellet melting).

- Performances (target criteria: cycle length about 480 EFPD (Equivalent Full Power Day), high fuel burn-up: $100 \mathrm{GWd} / \mathrm{t}$, and a zero or positive breeding gain).

The CFV core concept involves in its inner part a heterogeneous axial fuel column made of $\mathrm{UPuO}_{2}$ pellets with a thick fertile plate in the central zone. It is also characterized by an asymmetrical, crucible-shaped core with a sodium plenum above the fissile area (Fig. 2). 


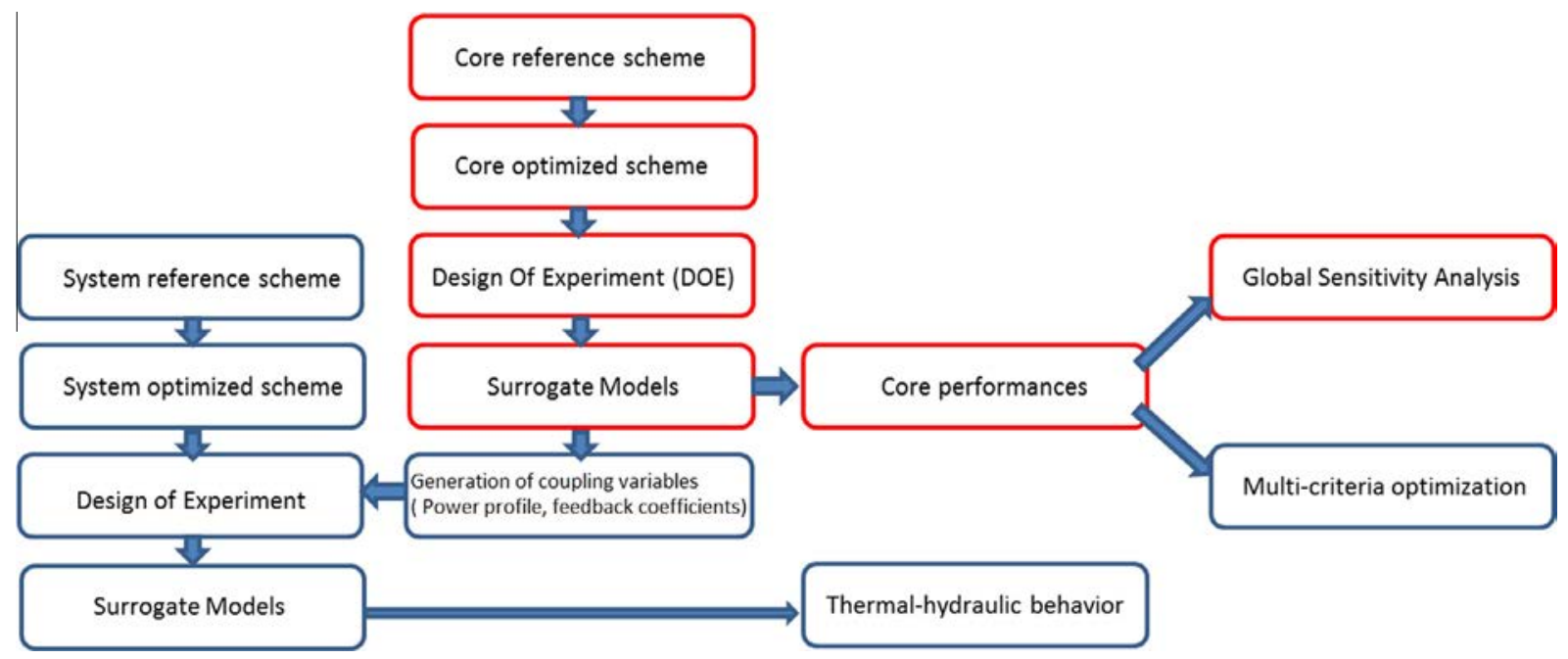

Fig. 1. Overview of the methodology sequence.

The CFV-v1 oxide core is composed of Inner (177 assemblies)/ Outer (114) core fuel assemblies, and three rings of reflectors (216 assemblies). Four additional rings are added for shielding (Fig. 3). Around 20 locations are foreseen for experimental subassemblies. The number of control/shutdown rods is respectively 12 and 6.

A first version of the CFV core, called CFV-v1, demonstrated the pre-feasibility of this concept. The CFV core, compared to previous SFR cores like SUPERPHENIX or EFR (European Fast Reactor) project, or to homogeneous standard SFR core, exhibit improvements in terms of performance and natural behavior.

Feedback coefficients are optimized (in particular sodium void coefficient) to allow better natural behavior in case of unprotected Loss of Flow Transients: no sodium boiling with enough margins (short term behavior), and temperature of neutron power shutdown reduced (long term behavior) compared to SUPERPHENIX core. These characteristics enable to eliminate energetic release severe accident scenarios for the CFV core in case of ULOF.

The overall negative sodium void coefficient (corresponding to combined core and plenum voiding) provides margin in case of global core boiling or sodium draining. This new specificity compared with standard core (void coefficient $>-5 \$$ ) can be extrapolated to large industrial core. The low core pressure drop is also

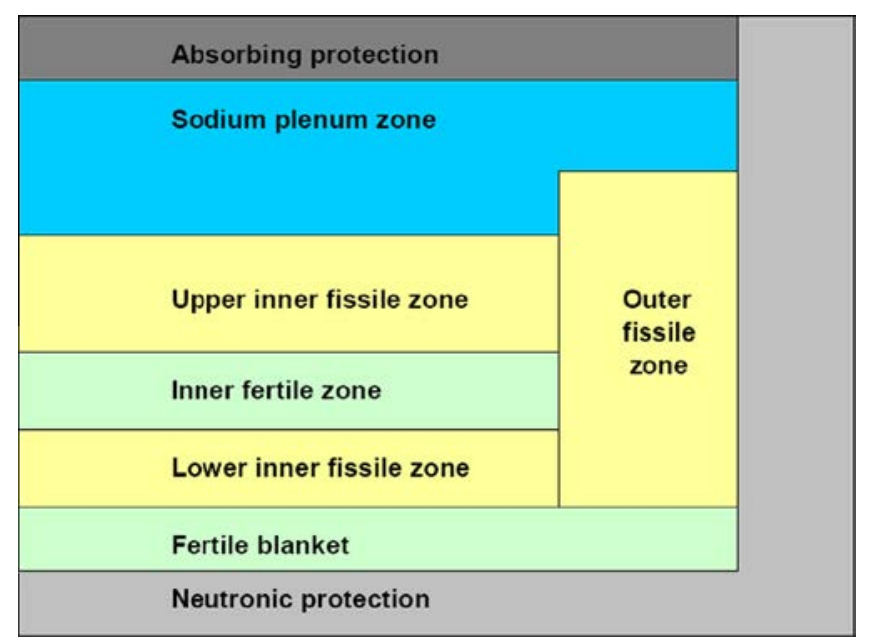

Fig. 2. Axial cross section of CFV core.

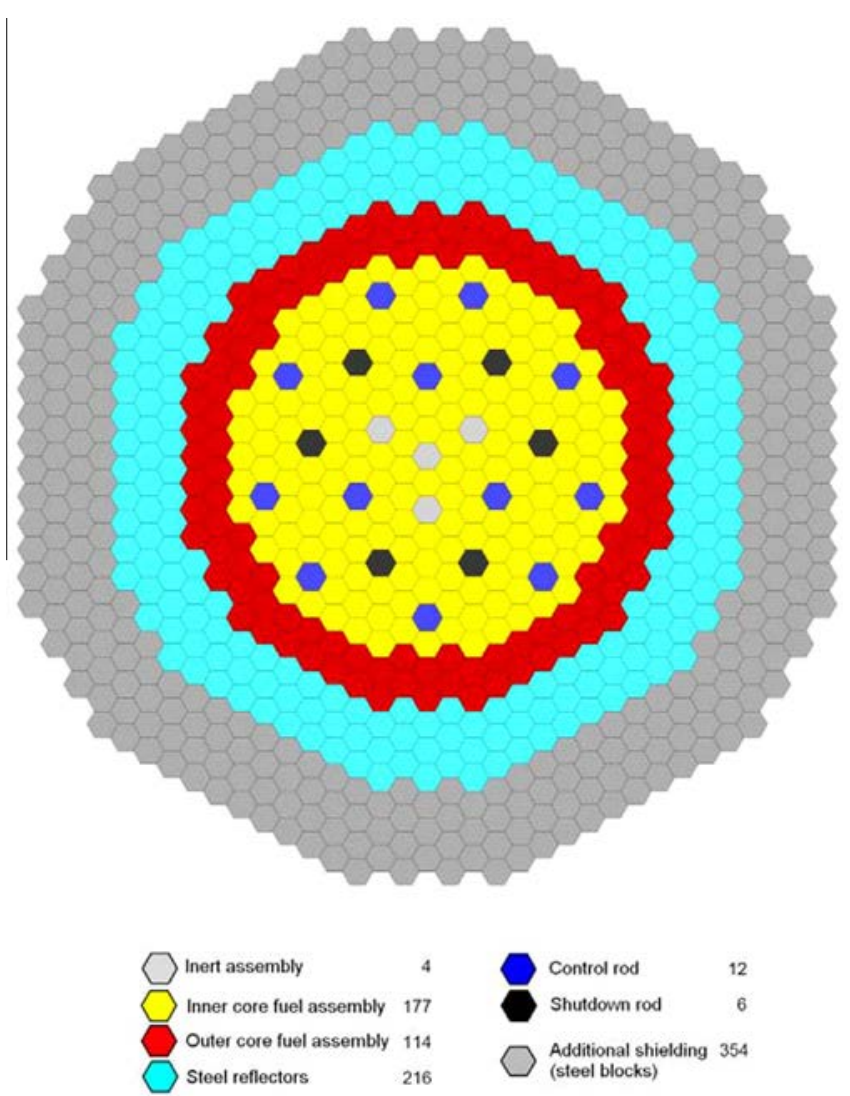

Fig. 3. Radial cross section of the CFV-v1 core.

favorable in case of rapid Loss of Flow Transient or in case of natural convection flow. The weak loss of reactivity of the core during irradiation cycle is also favorable in case of the Control Rod Withdrawal accident.

Analysis of severe accident conditions is under progress. Ongoing definition of an updated version of CFV core should lead to improve its natural behavior, with the objectives to increase the sodium boiling margin and to ensure a robust demonstration of no fuel melting in case of Control Rod Withdrawal situation. 
Table 1

Performances definitions of SFR core.

\begin{tabular}{|c|c|c|c|c|}
\hline Designation & Acronym & Definition & Objective & Classification \\
\hline $\begin{array}{l}\text { Beginning of Life Plutonium } \\
\text { inventory }\end{array}$ & IPU & Plutonium quantity needed to enable divergence & $\begin{array}{l}\text { To be } \\
\text { minimized }\end{array}$ & Costs \\
\hline Reactivity loss per cycle & DRHO & Loss of reactivity compensated by control rod withdrawal & Closest to zero & Safety \\
\hline $\begin{array}{l}\text { Minor Actinides production per } \\
\text { EFPD }\end{array}$ & MAeff & $\begin{array}{l}\text { Long lived high activity elements production by multiple neutron capture on } \\
\text { Plutonium isotopes }\end{array}$ & $\begin{array}{l}\text { To be } \\
\text { minimized }\end{array}$ & Durability \\
\hline Breeding gain & BG & Plutonium effective production during irradiation & $\begin{array}{l}\text { To be } \\
\text { maximized }\end{array}$ & Durability, costs \\
\hline Maximum Linear power & PLINmax & Maximum power generated in one centimeter of a fuel pin & $\begin{array}{l}\text { To be } \\
\text { minimized }\end{array}$ & Safety \\
\hline Clad DPA & DPAmax & Damage on stainless steel clad responsible for embrittlement & $\begin{array}{l}\text { To be } \\
\text { minimized }\end{array}$ & Safety \\
\hline $\begin{array}{l}\text { Sodium thermal expansion } \\
\text { feedback }\end{array}$ & NAFB & Reactivity change induced by sodium expansion & $\begin{array}{l}\text { To be } \\
\text { minimized }\end{array}$ & $\begin{array}{l}\text { Acceptability, } \\
\text { safety }\end{array}$ \\
\hline
\end{tabular}

\subsection{Model objectives and assumptions}

The main objective is to implement a deterministic calculation scheme suitable for the predesign and optimization process involving building up surrogate models. This step requires tens of thousands deterministic calculations in order to cover the whole range of performance variables.

\subsubsection{Simulation tools}

Two codes were used during this study. The simulations of the core neutronics behavior during irradiation were performed using the reference ERANOS calculation tool (European Reactor Analysis Optimized code System, Ruggieri et al., 2006), while the mathematical treatments (Design of Experiment, metamodels, Global Sensitivity Analysis) were done using the URANIE (Gaudier, 2010) platform.

ERANOS is a deterministic neutron physics code being developed by CEA for 25 years. Its different modules can cope with the specific physical phenomena involved in a fast reactor core. The ECCO module is use $d$ to perform cells calculation (computation of nuclear cross section), based on sub-groups method. The code resolves integral transport equation using a fine 1968 energygroup scheme for the self-shielding based on sub-group and probability table method, The nuclear data employed for these calculations are from the latest release of the JEFF3.1.1 European library. Boltzmann transport equation can then be solved in different geometries (1D, 2D or 3D) using different solvers for transport or diffusion computations. The resulting neutron fluxes can then be coupled with Bateman equation solver to perform depletion calculation all along the irradiation. The reference transport calculation is performed using BISTRO (2D) or VARIANT (3D) in a $\mathrm{S}_{8}-\mathrm{P}_{1}$ scheme. Moreover, ERANOS is designed for standard and generalized perturbation theories, allowing the calculation of sensitivity profiles for linear and bilinear functionals of the direct and adjoint fluxes.

URANIE is an uncertainty/sensitivity platform developed by CEA; it is based on the ROOT system (ROOT, 1998) an object oriented framework for large scale analysis and data mining. This platform includes several modules that allow performing: Design of Experiments techniques (LHD, quasi Monte-Carlo), surrogate models, spectral projection, sensitivity analysis, optimization (with OPT++ and VIZIR libraries), and computing distribution.

\subsubsection{Model objectives and simplified deterministic calculation scheme}

To achieve the implementation of a calculation scheme suitable for the predesign process, ERANOS datasets have to be automatically regenerated each time a new configuration is set, on one hand, and, on the other hand, the time spent in core calculation has to be as low as possible. New model predicts the most impor- tant neutronics performances of the CFV core (cf. Table 1): plutonium inventory, reactivity losses per irradiation cycle, minor actinide production, breeding gain, maximal values of linear power and damage, and void effect. These outputs, classified in performance, safety, or durability and acceptability, as requested by the Generation IV high priority list definition (GIF, 2012), were later optimized using surrogate models.

The plutonium inventory at the core BOL (Beginning of Life) is a parameter driving both cost and feasibility of the whole system; it should be as low as possible.

The minor actinide production, representing the effective quantity of long-lived wastes produced during irradiation cycle is a key feature regarding sustainability. In a SFR, transmutation is possible due to the hard neutron spectrum, and one of the objectives is to demonstrate actinides incineration feasibility.

Breeding gain measures the ability of a reactor to produce fissile isotopes while burning them. It expresses the excess (or loss if negative) of fissile material produced versus the consumed quantity (Waltar and Reynolds, 1981). The sustainability of a fast reactor fleet relies directly on this performance. As Gen-IV is intended to have a closed fuel cycle, the irradiation should produce enough fissile materials to allow refuelling of the same reactor core. The breeding gain is then null (so-called "zero breeding gain" target, Yang, 2012).

Reactivity loss per cycle is a performance indicator expressing the core controllability. During irradiation the fuel is depleted and thus the core intrinsic reactivity decreases. This loss is compensated by control rod (neutron absorber) withdrawal. Decreasing this loss diminishes control rods movements and thus the likelihood of an Unprotected Control Rod Withdrawal (UCRW) decreases. This performance is taken here as a safety indicator.

The maximum linear power and the maximal irradiation damage to the cladding are fuel element safety criteria estimators. The maximal linear power influences directly the temperature to fusion margin in the hottest fuel pin. The damage to the cladding is linked to the phenomenon of steel embrittlement; its minimization decreases the likelihood of a cladding rupture during operations.

Finally the sodium feedback coefficient is a major safety and acceptability issue of fast reactors systems. In a fast reactor core, the thermal expansion of the coolant leads to a harder neutron spectrum and thus to an increase of the reactivity. It is worth mentioning that this spectrum effect, in case of sodium voiding, is compensated by the neutron leakage effect (increase of the mean free path), which is very important especially in the ASTRID design. Lowering this feedback is then considered as an improvement in the safety, as it has a direct impact on both Na peak temperature and time to reach this peak in case of unprotected transient. 
Table 2

Core physics integral parameters.

\begin{tabular}{|c|c|c|c|c|c|}
\hline Performance & Unit & CFV-v1 & Simplified scheme & Absolute error & Reference scheme uncertainties \\
\hline Enrichments: Internal core/external core & $(-1-)$ & $23.52 / 20.02$ & $23.82 / 20.28$ & $0.3 / 0.26$ & $\mathrm{n} / \mathrm{a}$ \\
\hline Plutonium quantity at BOL & $\mathrm{kg}$ & 4927 & 4995 & 68 & $\mathrm{n} / \mathrm{a}$ \\
\hline$\beta_{\text {eff }} \mathrm{EOC}$ & $\mathrm{pcm}$ & 364 & 365 & 1 & 35 \\
\hline Breeding gain & - & -0.02 & -0.02 & 0 & 0.05 \\
\hline Cycle reactivity loss & $\mathrm{pcm}$ & -1500 & -1490 & 10 & $>300$ \\
\hline Thermal power: Internal core/external core & MW & $983 / 507$ & $989 / 497$ & $6 / 10$ & $>30 / 15$ \\
\hline Maximum linear power at BOC & $\mathrm{W} / \mathrm{cm}$ & $484 / 385$ & $436 / 385$ & $-48 / 0$ & $>24$ \\
\hline Maximum linear power at EOC & $\mathrm{W} / \mathrm{cm}$ & $460 / 344$ & $424 / 346$ & $-36 / 2$ & $>24$ \\
\hline Maximum cladding damage & DPA & 113 & 111 & 2 & 17 \\
\hline Minor actinides production per cycle & $\mathrm{kg}$ & 17.3 & 16.6 & 0.7 & 2.6 \\
\hline
\end{tabular}

\subsubsection{Model assumptions}

Once a core design is defined, a reference scheme, involving a high number of combined flux and depletion calculations, is applied to estimate the characteristics of the core all along its life time. With ERANOS, the 3D distribution of flux takes $48 \mathrm{~h}$ (reference scheme) to compute, and the code execution time is further increased in case of feedback coefficients evaluation. This modeling approach is not compatible with the predesign methodology based on surrogate models and genetic algorithms. To overcome these difficulties, a number of simplifications have been introduced, leading to a new, generic, and fast-running model. This model is based on the following assumptions:

- Numbers of sub-assemblies (inner and outer fuel assemblies, control rods, shutdown rods, steel reflectors and additional shielding) are fixed and their positions are invariant; the CFV$v 1$ configuration is taken as the reference. The main reason for this assumption is to avoid a calculation of the fuel reloading pattern for each configuration. The number of core refuelling batches is equal to 4 . After each irradiation cycle, one fourth of the fuel is renewed, a sub-assembly LifeTime (LT) is thus four times the irradiation duration.

- "Once through irradiation" hypothesis. Due to depletion phenomenon the fuel composition changes along the cycle. Away from core's Beginning of Life and core's End Of Life (EOL), the refuelling scheme brings regularly fresh fuel and withdraw irradiated one. After a certain number of batch refuelling, an equilibrium irradiation cycle will be reached. The beginning of this cycle (BOC) and its end (EOC) will be our main focus. The once through hypothesis allows assessing in a simplified way the core characteristics in this equilibrium state.

The underlying assumption consists in considering that each irradiation cycle is synonym of the same average amount of burn-up $b$. Then, knowing the core composition at the beginning of the cycle (Eq. (1.a)) and the end of cycle (Eq. (1.b)) one obtains for the total average burn-up of the core:

$B U_{B O C}=\frac{1}{4}(0 \cdot b+1 \cdot b+2 \cdot b+3 \cdot b)=\frac{3}{2} \cdot b=\frac{3}{8} \cdot B$

$B U_{E O C}=\frac{1}{4}(1 \cdot b+2 \cdot b+3 \cdot b+4 \cdot b)=\frac{5}{2} \cdot b=\frac{5}{8} \cdot B$

With $B$, the core total average burn-up of for the whole fuel lifetime. $B=4 \cdot b$.

The global composition of a core initially composed of only fresh sub-assembly, will be equivalent to an equilibrium core at an irradiation time of $\frac{3}{8} \cdot L T$ for BOC and $\frac{5}{8} \cdot L T$ for EOC.
- Both BOL fuel plutonium and diluted minor actinides isotopic compositions are kept to their reference value. Their values (Buiron et al., 2012) are taken from the up-to-date reference scenario of a fast reactor fuel cycle.

These assumptions lead to the implementation of a calculation scheme suitable to the predesign methodology. The main steps are listed below:

- For each configuration, full 3D hexagonal core geometry and its associated meshing are automatically generated.

- Then, the average Plutonium content in the core is searched. The target criterion is the critically of the core at the end of the equilibrium cycle. Several flux and depletion calculations are iteratively performed in diffusion theory. The iterations are driven by a Newton-Raphson algorithm, until the criticality criterion is met. For each iteration, new cross section calculations are done with the ECCO module of ERANOS.

- Once the average Plutonium content is determined, cross sections are re-computed and a complete irradiation calculation is performed with the VARIANT transport module of ERANOS for flux calculations associated with Bateman equation resolution for depletion. The control rod worth and their critical heights are estimated at the Beginning of Cycle.

- Finally, all the major feedbacks coefficients (Doppler coefficient, Sodium thermal expansion, Hexagonal Can, Cladding, Fuel and diagrid dilatations coefficients) are calculated in a simplified diffusion theory for both BOC and EOC.

The neutronics calculations CPU time has decreased from $48 \mathrm{~h}$ (reference scheme) to less than $8 \mathrm{~h}$ (predesign and optimizationmethodology-compatible scheme). Furthermore, the simulation results are compared with the reference scheme outputs and are in good agreement.

\subsection{Comparison with reference calculation scheme}

Tables 2 and 3 summarize the optimized scheme results, and compare them to the characteristics of the CFV-v1 core, as reported in Chenaud et al. (2013). Plutonium inventory, breeding gain, reactivity losses, and thermal power, are correctly estimated, as the error range is below the percent.

The local properties are slightly less well predicted, especially the maximum linear power (hot spot) of the core, for which the relative error goes up to $10 \%$. The absence of refueling pattern calculations, can explain this difference. The results associated with such local quantities should be then considered relatively, as global trends.

Results on feedback coefficients are satisfying, despite of the diffusion assumption. The errors bounds are roughly within the reference scheme calculations errors. The relative error generated 
Table 3

End of cycle feedback coefficients.

\begin{tabular}{|c|c|c|c|c|c|}
\hline Performance & Units & CFV-v1 & Simplified scheme & Relative error (\%) & Reference scheme uncertainties (\%) \\
\hline Doppler constant $K d$ & $\mathrm{pcm}$ & -1018 & -980 & -3.75 & 15 \\
\hline Sodium thermal expansion feedback & $\mathrm{pcm} /{ }^{\circ} \mathrm{C}$ & 0.093 & 0.112 & 19.5 & 20 \\
\hline Fuel thermal expansion feedback & $\mathrm{pcm} /{ }^{\circ} \mathrm{C}$ & -0.2272 & -0.2581 & -13.5 & 25 \\
\hline Cladding axial thermal expansion feedback & $\mathrm{pcm} /{ }^{\circ} \mathrm{C}$ & 0.06084 & 0.05688 & -6.5 & 20 \\
\hline Hexcan axial thermal expansion feedback & $\mathrm{pcm} /{ }^{\circ} \mathrm{C}$ & 0.01935 & 0.01678 & -13 & 20 \\
\hline Diagrid thermal expansion feedback & $\mathrm{pcm} /{ }^{\circ} \mathrm{C}$ & -0.8812 & -0.903 & -2.4 & 20 \\
\hline
\end{tabular}

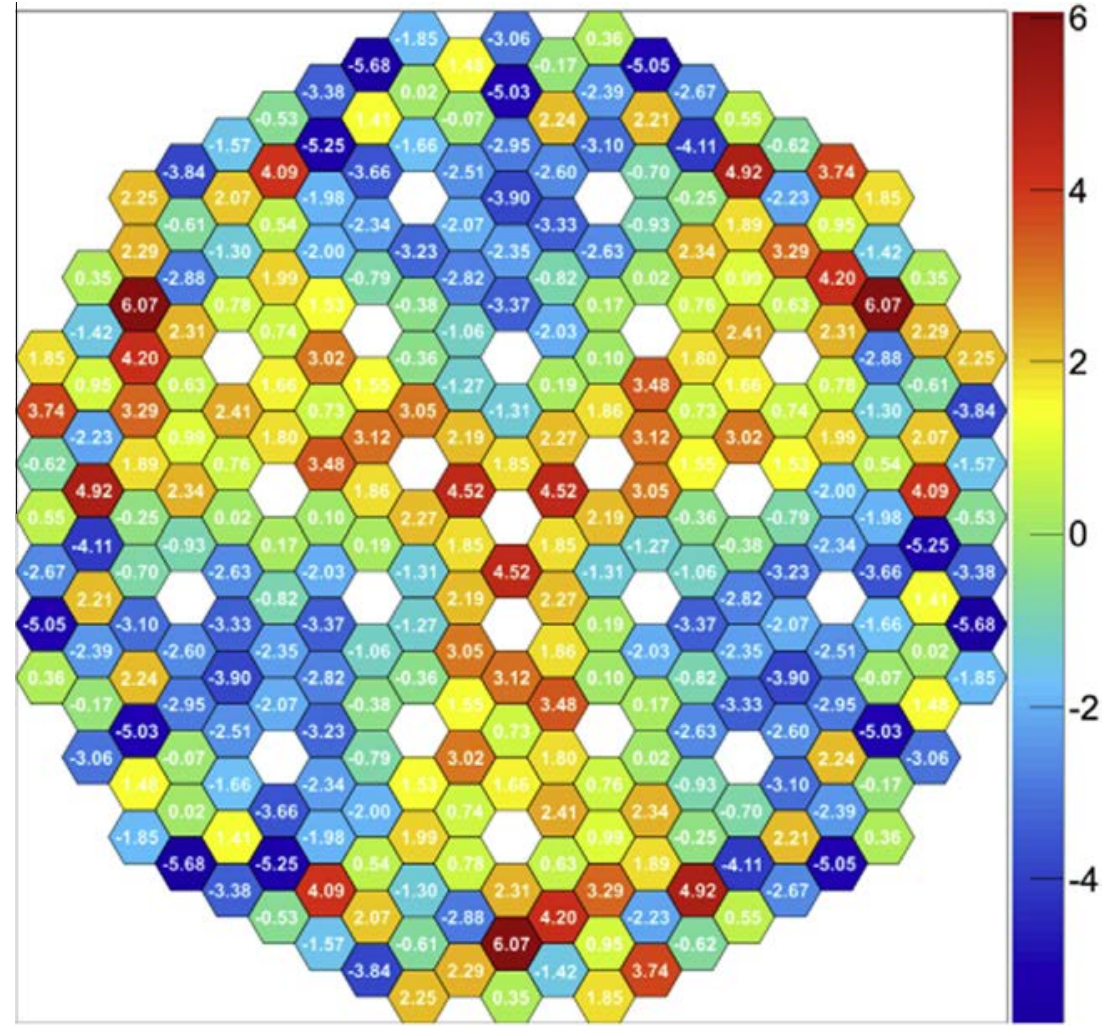

Fig. 4. Relative error on power each sub-assembly.

assembly per assembly is also quite satisfying, as it stands below $6 \%$ (see Fig. 4). However, those uncertainties are generally growing during the cycle, and Table 3 represents the upper bound uncertainties. Nevertheless, important biases remain (such as for the sodium void coefficient), and an a posteriori deterministic reference calculation of the optimized solution will be performed for further validation of the methodology. However, thermal expansion coefficients compensate each other during the transient so that the average behavior of the system is correctly reproduced using the simplified calculation scheme.

It will be shown in further studies that the overall system behavior during transients is mainly driven by the thermalhydraulics. The ongoing multi-objective study will demonstrate it.

\section{Design of Experiments and surrogate models development}

Even if the calculation time has been reduced to few hours in the previous step of the methodology, the complete application, including accurate global sensitivity analyses and multiobjectives optimization require more than a billion calculations (especially the computation of Sobol indexes is very consuming regarding the numbers of instances to run). For obvious reasons of computer resources this is still not suitable for the whole process. The following section describes the methods selected in order to be able to reproduce efficiently outputs from the code in a reasonable time, i.e. the optimized size limited sampling of the design space and the construction of accurate surrogate models.

\subsection{Parameters ranges}

The first step consists in defining the design space. Based on the design presented in Figs. 2 and 3, a set of design parameters has been specified (Table 4) along with their respective ranges.

Additional parameters are also included in the design space. The methodology aims at quantifying advantages and drawbacks of such design options. These additional parameters included increased heterogeneities in the fuel zones (different internal radii, enrichments), and the homogeneous dilution of minor actinides in the fuel. These parameters are summarized in Table 5. One ends up with 30 different design parameters, allowing a complete study of 
Table 4

Design parameters.

\begin{tabular}{|c|c|c|c|c|}
\hline Design parameters & Unit & Lower bound & Upper bound & Reference value \\
\hline External radius of fuel pellet & $\mathrm{cm}$ & 0.25 & 0.86 & 0.4225 \\
\hline Internal radius of fuel pellet & $\mathrm{cm}$ & 0.00 & 0.20 & 0.11 \\
\hline Thickness of the gap «pellet to cladding $\gg$ & $\mathrm{cm}$ & 0.0075 & 0.0325 & 0.0125 \\
\hline Thickness of the cladding & $\mathrm{cm}$ & 0.04 & 0.08 & 0.05 \\
\hline Spacer wire diameter & $\mathrm{cm}$ & 0.075 & 0.25 & 0.1 \\
\hline Wrapper pitch of spacer wire & $\mathrm{cm}$ & 15.0 & 25 & 18 \\
\hline Number of fuel pellet rings in a sub-assembly & - & 6 & 12 & 9 \\
\hline Thickness of the Hexcan & $\mathrm{cm}$ & 0.30 & 0.6 & 0.45 \\
\hline Thickness of the inter sub assembly sodium layer & $\mathrm{cm}$ & 0.30 & 0.6 & 0.45 \\
\hline Length of the upper gas plenum & $\mathrm{cm}$ & 70 & 110 & 93.3 \\
\hline Length of the fertile blanket & $\mathrm{cm}$ & 5 & 35 & 30 \\
\hline Length of the fertile slab & $\mathrm{cm}$ & 5 & 40 & 20 \\
\hline Length of the lower fissile zone & $\mathrm{cm}$ & 10 & 40 & 25 \\
\hline Length of the upper fissile zone & $\mathrm{cm}$ & 10 & 40 & 35 \\
\hline Importance of the diabolo shift & $\mathrm{cm}$ & 1 & 20 & 10 \\
\hline Length of the upper gas plenum & $\mathrm{cm}$ & 5 & 15 & 7.5 \\
\hline Length of the sodium plenum & $\mathrm{cm}$ & 30 & 60 & 40 \\
\hline Length of the upper neutronics protection & $\mathrm{cm}$ & 70 & 150 & 89.9 \\
\hline Enrichment ratio inner/outer core & - & 0.66 & 1.33 & 1.175 \\
\hline Porosity of the fuel & - & 0.94 & 0.97 & 0.955 \\
\hline Duration of an irradiation cycle & EFPD & 300 & 450 & 360 \\
\hline
\end{tabular}

Table 5

Additional design parameters.

\begin{tabular}{|c|c|c|c|c|}
\hline Conception parameters & Unit & Lower bound & Upper bound & Reference value \\
\hline Internal radius of fertile blanket pellets & $\mathrm{cm}$ & 0.0 & 0.2 & 0.11 \\
\hline Internal radius of fertile slab pellets & $\mathrm{cm}$ & 0.0 & 0.2 & 0.11 \\
\hline Internal radius of lower fissile pellets & $\mathrm{cm}$ & 0.0 & 0.2 & 0.11 \\
\hline Internal radius of upper fissile pellets & $\mathrm{cm}$ & 0.0 & 0.2 & 0.11 \\
\hline Internal radius of outer fissile pellets & $\mathrm{cm}$ & 0.0 & 0.2 & 0.11 \\
\hline Enrichment ratio between inner lower/outer core & - & 0.66 & 1.33 & 1.175 \\
\hline Enrichment ratio between inner upper/outer core & - & 0.66 & 1.33 & 1.175 \\
\hline Enrichment ratio between fertile blanket/outer core & - & 0 & 0.15 & 0 \\
\hline Enrichment ratio between fertile slab/outer core & - & 0 & 0.15 & 0 \\
\hline Percentage of Minor actinide in fresh fissile pellets & $\%$ & 0 & 7 & 0 \\
\hline Percentage of Minor actinide in fresh fertile pellets & $\%$ & 0 & 10 & 0 \\
\hline
\end{tabular}

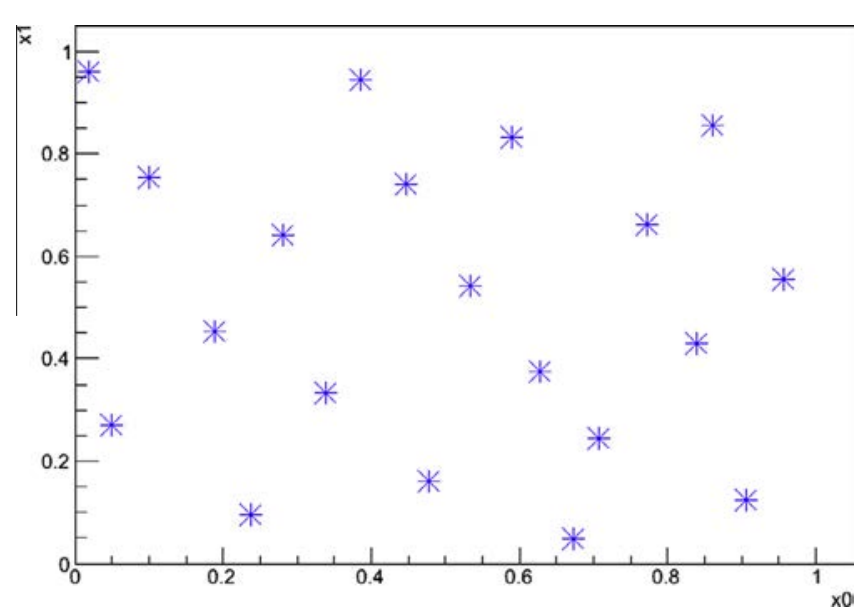

Fig. 5. Wrap around discrepancy optimized LHD; $d=2 ; N=20$.

the design. Those design parameters required 5200 ERANOS calculations to build the learning database.

\subsection{DOE technique used in the context of this work}

A standard method to cover a high dimensional space is the LHD (Latin Hypercube Design). Indeed, the intrinsic features of LHD sampling are that their size $N$ is independent from the number of dimensions; each dimension is being evenly sampled in $N$ different values. This last characteristic often leads in the literature to refer LHD as "space filling" designs.

However, basic LHD samplings can be ill-conditioned. An algorithm has been implemented in the methodology to insure their quality. The goal is optimize a criterion, representative of the space-covering quality of the design.

The chosen criterion is the wrap-around $W^{2}$ discrepancy (Eq. (2)), a statistical quantity measuring the distance of a distribution from a uniform one.

Iooss et al. (2010) pointed out good resistance of $W^{2}$-optimized LHD from a dimension reduction and their potential as bases for metamodel constructions.

$W^{2}=\left(\frac{4}{3}\right)^{d}+\frac{1}{N^{2}} \sum_{i, j=1}^{N} \prod_{k=1}^{d}\left[\frac{3}{2}+\left|x_{k}^{j}-x_{k}^{i}\right| \cdot\left(1-\left|x_{k}^{j}-x_{k}^{i}\right|\right)\right]$

where $k$ is the index over the dimension $d, i$ and $j$ the indices over the points (total number $N$ ). $x_{k}^{i}$ is the value of projection of vector $\vec{x}$ on the axis $k$.

The optimization algorithm developed is of simulatedannealing type. A scrambled random LHD design of size $N$ is produced in the hypercube space $[0 ; 1]^{d}$ (in this study $d=30$, the number of design parameters considered). This design is slightly modified step-by-step by randomly swapping two coordinates of two of its points. At each step, the wrap-around discrepancy is computed and the modification is either accepted or rejected 
following the "cooling schedule" of a simulated annealing algorithm (Kirkpatrick et al., 1983). Once convergence is reached, the resulting DOE is un-normalized to the real design space.

An example of the Design of Experiment produced by this method in two dimensions $(d=2$ and $N=20)$ is reproduced in Fig. 5.

Once the Design of Experiment is set, the URANIE CEA's platform is used to launch a neutronics calculation with the ERANOS code for each of the points in the design. Each point in the DOE is associated with a vector of value for design parameters, and script generates automatically the associated data file, calculations are then launched in multi-thread on a cluster.

Previous studies on simplified geometry, not reported here, showed that few hundred calculations were enough to ensure good metamodels in a 10 dimensions space. In our case, more than five thousands initial points (5200) have been calculated.

\subsection{Surrogate models buildup}

The constitution of the training database leads to the construction metamodels. The goal is to find a regression function able to accurately reproduce outputs from the calculations in the whole design space. For regression model, Artificial Neural Networks have been selected in this study for several reasons:

- The number of assumptions (smoothness, regularity, monotony, significant variables...) on the response function must be as low as possible.

- ANNs are considered as theoretically universal regression models.

- Their parameters computation is not time consuming.

The following section describes the structure chosen for the neural networks used. The one hidden layer structure used is shown in Fig. 6.

Each of the neuron of the network is an aggregative function of the form (Eq. (3)).

$\left.y(\vec{x})=g \sum_{i=1}^{d} w_{i} x_{i}+\beta\right)$ with $g(\tau)=\frac{1}{1+e^{-\tau / T}}$

The set of factors $\left\{w_{i} ; \beta ; T\right\}$ for each neuron are the parameters of the regression function adjusted in order to build a correct surrogate model. $g$ is called the activation function, $w_{i}$ are its weights, $\beta$ is its bias and $T$ its slope parameter. Several numbers of neurons in the hidden layers $\left(N_{h}\right)$ have been tested, for each output, the one leading to the best metamodel (in term of prediction coefficient $\mathrm{cf}$. Eq (5)) has been selected. $N_{h}$ range found is $[4 ; 9]$.

With such architecture for $N_{h}$ hidden neurons, the number of parameters $N_{p}$ of the metamodel is shown in Eq. (4). Range for $N_{p}$ is then $[158 ; 318]$. The network characteristics are shown in Table 6 .

$N_{p}=N_{h}(d+3)+2$

A so-called feed-forward algorithm implemented in URANIE platform was applied to each neural network to set the optimal value of other parameters.

\subsection{Quality assessment}

The choice of the method used to validate the built metamodels was motivated by two criteria:

- The method should give unbiased estimations of the metamodels quality. The accuracy criteria retained in this method is the correlation coefficient $Q^{2}$ (Eq. (5)), expressing how the meta- model outputs are close to the code responses. The maximum error MAX (Eq. (6)), expressing the local quality of the function, is also computed.

- If the quality criteria are not met, the method should drive an easy improvement of the metamodels.

$Q^{2}=1-\frac{\sum_{i=1}^{N}\left(y_{i}-\tilde{y}_{i}\right)^{2}}{\sum_{i=1}^{N}\left(\bar{y}-y_{i}\right)^{2}}$

$M A X=\max \left(\left|y_{i}-\tilde{y}_{i}\right|\right)$

where $y_{i}$ stands for the real model output at point $x_{i}, \tilde{y}_{i}$ the metamodel prediction at this point; and $\bar{y}$ the mean of all the $y_{i}$.

For these reasons, the choice of validating the metamodels with a second database has been made. Lin (2004) pointed out it was the only way to insure the absence of bias in a validating process. However, its main drawback is the additional cost induced by calculations. The information improvement brought by this second Design of Experiment should thus be optimized. This is done by minimizing the bias in quality estimations with a minimum number of points. The selected method follows the same approach as the previous one. The goal is to find in the design space the points that will minimize the discrepancy of the initial Design of Experiment.

$N_{c}$ candidates (in our study 3000) points are first generated following a quasi-Monte Carlo, low discrepancy sequence (Hammersley's one). Then points of the validation database are sequentially selected among these candidates. The selection criterion is the minimization of the $W^{2}$ discrepancy differences between the initial and the new design.

An example in two dimensions $(d=2, N=20$ for both training and validation) is given in Fig. 7. The blue stars are from the initial training database, and the red diamonds are from the subsequent validation database.

This method aims at selecting the furthest validation points from the whole training database. One aims by this way at capturing the right estimation of the prediction coefficient $Q^{2}$ and an upper bound for the maximum error.

To further improve the metamodels quality, an additional iterative step is performed. The main objective is to increase the local accuracy of the surrogates, where the error is found to be maximal. As the error is computed for each point of the validation database, one aims at reducing it in the problematic areas. For each metamodel, one simultaneously updates both training and validations database in the following manner, represented in Fig. 8.

- $N_{v}$ validation database points for which absolute error is maximal are identified and selected.

- These points are transferred in the training database.

- For each point transferred, two are added to the validation database. Locations of these two new points are in the neighborhood of the transferred one.

- A new set of metamodels parameters are searched to fit the new database, and are tested on the new validation database.

- This step is repeated sequentially until all quality criteria are met (in our case $Q^{2}>95 \%$ ).

Three thousands additional ERANOS calculations were done to constitute this validation database. For each metamodel, the iterative process described above has been applied twice, adding $N_{v}=$ 25 points at each iteration (100 in total for each metamodel, 50 in each database). Only the metamodel of sodium feedback coefficient required four iterations. In total (training and validation databases), 8200 ERANOS calculations were launched. 


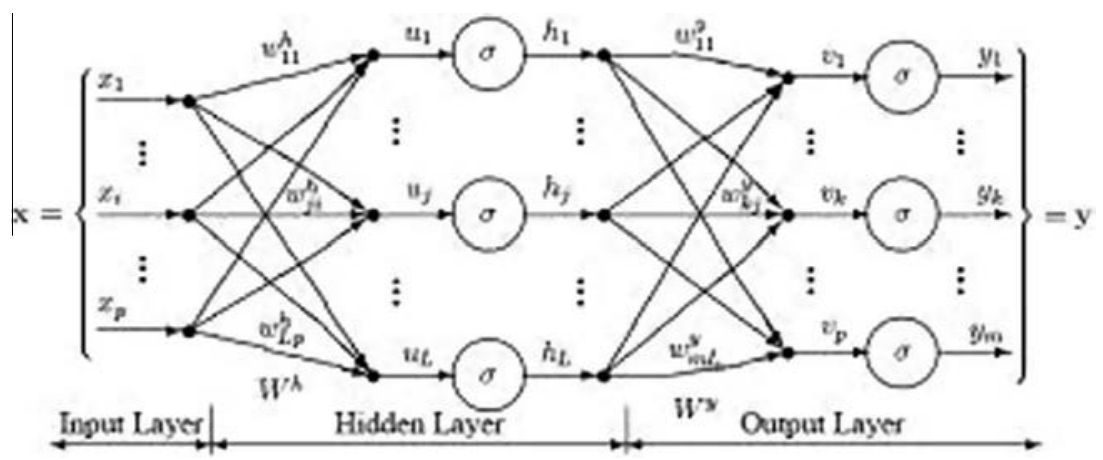

Fig. 6. One hidden layer neural network structure (from Reid (2014)).

Table 6

Metamodels quality estimators.

\begin{tabular}{|c|c|c|c|c|c|c|}
\hline Modeled response & Unit & $\begin{array}{l}\text { ANN hidden } \\
\text { neurons } N_{h}\end{array}$ & $\begin{array}{l}\text { ANN \# of } \\
\text { parameters } N_{p}\end{array}$ & $\begin{array}{l}Q^{2}(\%) \text { "goodness } \\
\text { of fit" }\end{array}$ & $\begin{array}{l}\text { MAX discrep. on } \\
\text { surrogate }\end{array}$ & $\begin{array}{l}\text { Reference calculation } \\
\text { uncertainties }\end{array}$ \\
\hline Plutonium Inventory & $\mathrm{kg}$ & 5 & 167 & 99 & 75 & $\mathrm{n} / \mathrm{a}$ \\
\hline Reactivity Loss & $\mathrm{pcm}$ & 5 & 167 & 99 & 400 & $>300$ \\
\hline Breeding Gain & $\%$ & 9 & 299 & 99 & 0.02 & 0.05 \\
\hline $\begin{array}{l}\text { Sodium dilatation feedback } \\
\text { coefficient }\end{array}$ & $\begin{array}{l}\mathrm{pcm} / \\
{ }^{\circ} \mathrm{C}\end{array}$ & 6 & 200 & 94 & 0.04 & 0.019 \\
\hline $\begin{array}{l}\text { Minor actinides effective } \\
\text { production rate }\end{array}$ & $\begin{array}{l}\mathrm{kg} / \\
\text { EFPD }\end{array}$ & 8 & 266 & 99 & 0.005 & 0.007 \\
\hline $\begin{array}{l}\text { Maximal damage on inner core } \\
\text { cladding }\end{array}$ & DPA & 5 & 167 & 97 & 6 & 15 \\
\hline $\begin{array}{l}\text { Maximal damage on outer core } \\
\text { cladding }\end{array}$ & DPA & 6 & 200 & 97 & 5 & 15 \\
\hline $\begin{array}{l}\text { Maximal linear power in inner } \\
\text { core at BOC }\end{array}$ & $\mathrm{W} / \mathrm{cm}$ & 7 & 233 & 97 & 37 & $>20$ \\
\hline $\begin{array}{l}\text { Maximal linear power in inner } \\
\text { core at EOC }\end{array}$ & $\mathrm{W} / \mathrm{cm}$ & 7 & 233 & 97 & 32 & $>20$ \\
\hline $\begin{array}{l}\text { Maximal linear power in outer } \\
\text { core at BOC }\end{array}$ & $\mathrm{W} / \mathrm{cm}$ & 7 & 233 & 96 & 29 & $>20$ \\
\hline $\begin{array}{l}\text { Maximal linear power in outer } \\
\text { core at EOC }\end{array}$ & $\mathrm{W} / \mathrm{cm}$ & 9 & 299 & 99 & 30 & $>20$ \\
\hline
\end{tabular}

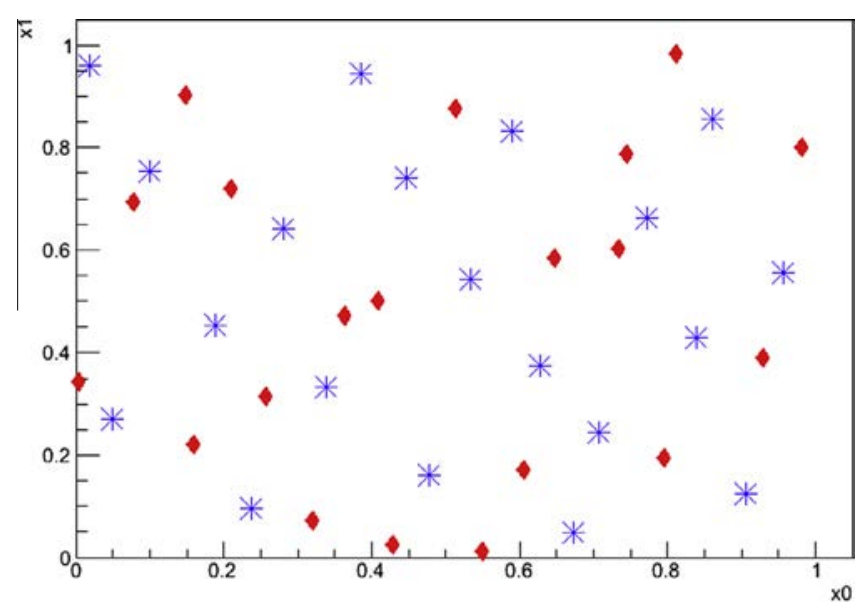

Fig. 7. Validation database in $2 \mathrm{D}$

Despite these extra calculations the sodium feedback metamodel was still below the quality criterion. A hypothesis to explain this is the numerical noise coming from the simplified calculation scheme on this output. Further investigation should be performed on this point.

Results of the 11 metamodels needed for representing the 7 performance parameters of this study are detailed in Table 6, along with the ANN parameters.
Locally, most of the discrepancies are within reference uncertainty margins. However, maximum linear power and reactivity loss exceed those margins, even if the correlation/quality factor is above $90 \%$. Nevertheless, they remain acceptable to perform a first exploratory trend analysis in an early design phase.

\section{Global Sensitivity Analysis}

\subsection{Computation of Sobol indices}

The Global Sensitivity Analysis framework has then been used to perform an in-depth study of the relationship between input parameters and the variance of the output models. These techniques are usually employed in an uncertainty framework. On the contrary here, they are used to determine and rank the input variables according to their influence on the model outputs. To get a thorough study, the Sobol first $S_{i}$ (Eq. (7)) and total $S_{T i}$ (Eq. (8)) order indices have been computed (Saltelli et al., 2010). The goal of this computation is to provide designer with a multicriteria analysis tool, by highlighting the important design parameters of the studied concept.

$S_{i}=\frac{V_{x_{i}}\left(E_{x \sim i}\left(y \mid x_{i}\right)\right)}{V(y)}$

$S_{T i}=1-\frac{V_{x \sim i}\left(E_{x i}\left(y \mid x_{\sim i}\right)\right)}{V(y)}$ 


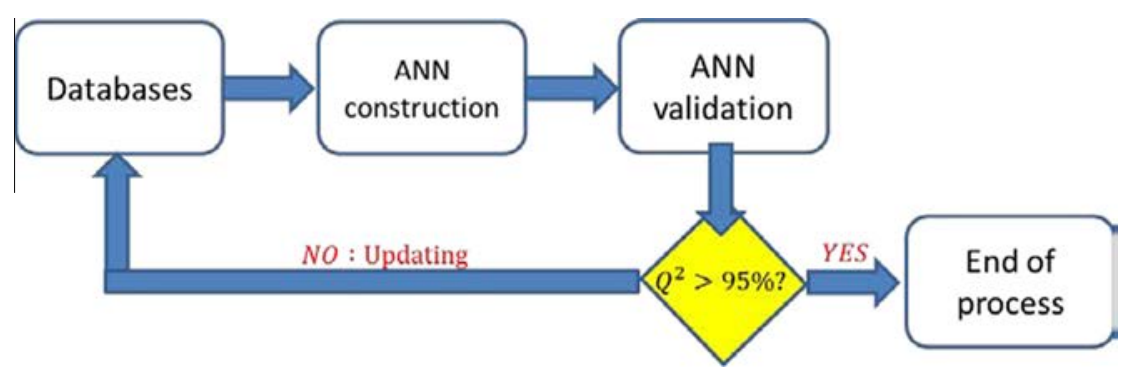

Fig. 8. Iterative ANN construction.

Table 7

First Sobol indices of influent variables.

\begin{tabular}{|c|c|c|c|c|c|c|c|}
\hline Variable & $\begin{array}{l}\text { Pu inventory } \\
\text { (IPU) }\end{array}$ & $\begin{array}{l}\text { MA production } \\
\text { (MAeff) }\end{array}$ & $\begin{array}{l}\text { Reactivity Loss/cycle } \\
\text { (DRHO) }\end{array}$ & $\begin{array}{l}\text { Max linear power } \\
\text { (PLINmax) }\end{array}$ & $\begin{array}{l}\text { Max DPA } \\
\text { (DPAmax) }\end{array}$ & $\begin{array}{l}\text { Breeding gain } \\
\text { (BG) }\end{array}$ & $\begin{array}{l}\text { Sodium feedback } \\
\text { (NAFB) }\end{array}$ \\
\hline Number of fuel rings & -66 & +4.2 & +33 & +81 & +56 & +16 & $\mathrm{X}$ \\
\hline Pellet outer radius & -21 & +13 & +44 & $\mathrm{X}$ & +28 & +58 & -7.2 \\
\hline Fertile Slab Height & -1.6 & $\mathrm{X}$ & $\mathrm{X}$ & $\mathrm{X}$ & +2.8 & $\mathrm{X}$ & -8.8 \\
\hline Lower Fissile Height & -1.5 & $\mathrm{X}$ & +3.0 & +4.9 & $\mathrm{X}$ & +4.6 & -31 \\
\hline Upper Fissile Height & -1.3 & $\mathrm{X}$ & +2.5 & +3.8 & $\mathrm{X}$ & +3.0 & -23 \\
\hline Spacer Wire Thickness & -1.3 & $\mathrm{X}$ & $\mathrm{X}$ & $\mathrm{X}$ & $\mathrm{X}$ & $\mathrm{X}$ & $\mathrm{X}$ \\
\hline $\begin{array}{l}\text { MA content diluted in } \\
\text { fissile zones }\end{array}$ & $\mathrm{X}$ & +54 & $\mathrm{X}$ & $\mathrm{X}$ & $\mathrm{X}$ & +3.6 & -4.6 \\
\hline $\begin{array}{l}\text { MA content diluted in } \\
\text { fertile zones }\end{array}$ & $\mathrm{X}$ & +16 & $\mathrm{x}$ & $\mathrm{X}$ & $\mathrm{X}$ & $\mathrm{X}$ & $\mathrm{X}$ \\
\hline Cycle length & $\mathrm{X}$ & $\mathrm{X}$ & -1.4 & $\mathrm{X}$ & -4.0 & $\mathrm{X}$ & $\mathrm{X}$ \\
\hline Fertile blanket height & $\mathrm{X}$ & $\mathrm{X}$ & $\mathrm{X}$ & +1.9 & $\mathrm{X}$ & +4.2 & -3.9 \\
\hline
\end{tabular}

With $\left[V_{x_{i}} ; E_{x i}\right]$ are standing respectively for variance and mean taken over $x_{i}$, and $\left[V_{x \sim i} ; E_{x \sim i}\right]$ for variance and mean taken over all factor except $x_{i}$.

The first order index is a normalized value (between 0 and 1) expressing the percentage of variance of the output $y$ explained by the variable $x_{i}$. The total order includes, along with the former, the percentage of variance due to the interaction of $x_{i}$ with all the other variables.

The Sobol indices values are then used along with twodimensional plots of the output functions to complete the results with the trends of variation.

An example of results of a complete Global Sensitivity Analysis is presented in Appendix A. In the following Table 7, the influent variables are highlighted:

- The value indicates the first Sobol index (given in \%).

- Plus and minus signs represent trends: "+" (resp. “-") means that an increase of the design parameter leads to an improvement (resp. a drop) of the performance.

- A " $X$ " sign is used when the variable influence is negligible (below the percent).

Among the 30 initials variables, 10 of them are found to have a significant influence on the performances. The number of fuel rings per sub-assembly and the outer pellet radius appears to be influent in most of the performances. The large changes induced by these variables on the core radial size explain this phenomenon. The Plutonium inventory is directly driven by the volume of fuel in the core and the minimization of this performance leads to small core. On the contrary quantities such as breeding gain, reactivity loss and minor actinide production are dependent on the efficiency in the neutron use. Indeed as the core size increase, the proportion of neutron leakage tends to decrease and thus neutrons "saved" can breed the fertile material, or transmute minor actinides.
Initial content of minor actinides diluted in the core is the main parameter influencing the effective nuclear waste production. The possibility to design a "minor actinide burner" is then mainly affected by the choice to dilute these actinides in the fuel. This impacts the whole fuel processing and managing strategy. This investigation (especially regarding costs) is beyond the present exploratory study.

The fuel element safety estimators (maximal power and damage) are impacted by the number of pin in each sub-assembly. As the global thermal power is fixed (1500 MWth) the increase in the number of fuel element involves a decrease in the neutron flux in each individual one. As the neutron flux is the phenomenon responsible for the power and damage generated in a pin, these safety estimator are improved.

The global sodium feedback coefficient value is mainly driven by the axial size of the core. While the sodium is dilating, two antagonist phenomena occur:

- The neutron spectrum is hardening, leading to an increase in the reactivity.

- The loss of moderation increases the leakage of neutrons outside of the core, leading to a negative contribution to the feedback.

A GSA on this performance outlines the importance of the later while designing a low void effect core. A decrease in the core size is preferable to improve the leakage contribution. The main influent variables are those of the axial fuel element size. This can be explained by the presence of a sodium plenum and a "diabolo shift" above the fuel zone, enhancing the efficiency of axial leakage in this zone.

The GSA allows also determining which performances will be antagonist for the design of the reactor. Typically looking at the results concerning plutonium inventory and reactivity loss (first 
Table 8

Configuration with enhanced safety estimators.

\begin{tabular}{|c|c|c|c|}
\hline Design parameters & Units & Values & CFV-v1 reference value \\
\hline Number of fuel pellet rings in a sub-assembly & - & 12 & 9 \\
\hline External radius of fuel pellet & $\mathrm{cm}$ & 0.36 & 0.42 \\
\hline Internal radius of fuel pellets & $\mathrm{cm}$ & 0.0 & 0.11 \\
\hline Spacer wire diameter & $\mathrm{cm}$ & 0.07 & 0.10 \\
\hline Duration of irradiation cycle & EFPD & 300 & 360 \\
\hline Enrichment ratio inner lower/outer core & - & 1.33 & 1.17 \\
\hline Enrichment ratio inner upper/outer core & - & 1.00 & 1.17 \\
\hline Performances & Units & Values & CFV-v1 reference value \\
\hline Plutonium inventory & $\mathrm{kg}$ & 6150 & 4900 \\
\hline MA production & kg/EFPD & 0.06 & 0.05 \\
\hline Reactivity loss per cycle & $\mathrm{pcm}$ & -1100 & -1500 \\
\hline Max linear power & $\mathrm{W} / \mathrm{cm}$ & 315 & 480 \\
\hline Max DPA & DPA & 90 & 120 \\
\hline Breeding gain & - & 0.01 & -0.02 \\
\hline Sodium feedback & $\mathrm{pcm} /{ }^{\circ} \mathrm{C}$ & -0.03 & 0.09 \\
\hline
\end{tabular}

Table 9

"Sustainable" configuration.

\begin{tabular}{|c|c|c|c|}
\hline Design parameters & Units & Values & CFV-v1 reference value \\
\hline Number of fuel pellet rings in a sub-assembly & - & 8 & 9 \\
\hline External radius of fuel pellet & $\mathrm{cm}$ & 0.43 & 0.42 \\
\hline Percentage of Minor actinide in fresh fissile pellets & $\%$ & 3.5 & 0 \\
\hline Percentage of Minor actinide in fresh fertile pellets & $\%$ & 5.0 & 0 \\
\hline Enrichment ratio inner lower/outer core & - & 1.10 & 1.17 \\
\hline Enrichment ratio inner upper/outer core & - & 1.10 & 1.17 \\
\hline Length of the lower fissile zone & $\mathrm{m}$ & 20 & 25 \\
\hline Length of the fertile slab & $\mathrm{cm}$ & 27.5 & 20 \\
\hline Performances & Units & Values & CFV-v1 reference value \\
\hline Plutonium inventory & $\mathrm{kg}$ & 4350 & 4900 \\
\hline MA production & $\mathrm{kg} / \mathrm{EFPD}$ & -0.188 & 0.05 \\
\hline Reactivity loss per cycle & $\mathrm{pcm}$ & -1600 & -1500 \\
\hline Max linear power & $\mathrm{W} / \mathrm{cm}$ & 580 & 480 \\
\hline Max DPA & DPA & 140 & 120 \\
\hline Breeding gain & - & 0.03 & -0.02 \\
\hline Sodium feedback & $\mathrm{pcm} /{ }^{\circ} \mathrm{C}$ & 0.20 & 0.09 \\
\hline
\end{tabular}

and third columns) one observes that most of the influent variables are acting in opposite way on these two performances.

\subsection{Alternative configurations}

Metamodels and sensitivity analysis results can then be used to efficiently predict performances of new core configurations. We exhibit here two alternative core configurations, the Sobol indices of Table 7 and 3D curves (as presented in Appendix A) allowed to guide this predesign process according to different objectives:

- First configuration objectives were to simultaneously enhance four safety estimators (PLINmax, DPAmax, DRHO, NAFB) compared to the CFV-v1 reference target;

- Second configuration objective was to design a "sustainable" configuration (i.e., a reduced plutonium inventory IPU and minor actinides production MAeff along with a positive breeding gain $\mathrm{BG}$ ).

The characteristics of the two core configurations are detailed in Tables 8 and 9. Performances in green indicate an improvement compared to CFV-v1 values, whereas red indicates a worsening.

The first configuration has been obtained by increasing the number of fuel pins in a sub-assembly, and reducing their internal and external radiuses, in order to achieve low linear power and damage to the cladding. Spacer wire diameter has been reduced to its minimum value to keep the sub-assembly pitch in a reasonable value $(20 \mathrm{~cm})$. Irradiation cycle length has been reduced in order to minimize the reactivity loss during cycle. Finally, enrichment ratios have been chosen in order to increase the neutron leakage in the upper part of the core and thus enhance the sodium reactivity feedback coefficient.

The second configuration has been obtained by reducing the number of fuel pins in a sub-assembly and increasing their diameter. This allowed achieving a lower plutonium inventory. Dilution of minor actinides in both fissile and fertile fuel zones lead to a net negative yield of these elements and a reasonable reactivity loss during the cycle. Finally, fuel zones height and enrichment were modified in order to reduce the maximum linear power and dose (DPA).

\section{Multi-Objective Optimizations}

To complement the decision helping methodology described in the previous paragraph, the analysis of Multi-Objective Optimization $(\mathrm{MOO})$ results is presented here. The goals of this step are:

- To identify optimal configurations: In the MOO framework, one retains the Pareto definition of optimality: i.e., a configuration is optimal if there is no other configuration improving simulta- 
neously all its performances. A configuration is optimal when the gain on a performance involves necessarily losses in at least one other performance. Thus a whole set of Pareto optimal solutions or "Pareto Front" has to be found. The retained configuration by the decision makers should belong to this set.

- To highlight overall trends: As the optimal solutions constitute a set, some essential statistical information can be extracted from it. In particular, interactions between performances, expressing the trade-off, are relevant in the decision making process by identifying what will be the drawbacks of improving a specific performance.

\subsection{Optimization perimeter}

As depicted in Table 1, the performed optimization exercise consists in maximizing the effective breeding gain and minimizing the following quantities:

- The Plutonium inventory.

- The effective production of minor actinides.

- The absolute value of reactivity loss per cycle.

- The maximum linear power.

- The maximum dose to the cladding in the core.

- The sodium feedback coefficient.
The optimization problem was also constrained as follows:

- Maximum dose on the cladding has to be lower than $150 \mathrm{dpa}$ : the studied concept is characterized by AIM1 steel cladding for which a too high dose is synonymous of high probability of cladding failures.

- Reactivity loss has to be greater than $-2000 \mathrm{pcm}$ : beyond this limit, the control rods movement would be too important, leading to safety concerns.

- Breeding gain absolute value should be lower than 0.05: One of the Gen-IV objectives being to build a reactor close to isogeneration.

- Maximum linear power should be between 325 and $625 \mathrm{~W} / \mathrm{cm}$ : a too high value means a too narrow margin to fuel melting in nominal condition. Too low values are also forbidden due to the lower conductivity in the absence of a fuel-cladding bond.

- Sodium feedback coefficient has to be lower than $0.25 \mathrm{pcm} /{ }^{\circ} \mathrm{C}$ : the value of this positive feedback coefficient should be limited for acceptability and safety concerns.

- Sub-assembly pitch should be lower than $20 \mathrm{~cm}$ : A higher value would lead to too large cores.

- The ratio $\frac{\text { External diameter of fuel pin }}{\text { Cladding thickness }}$ should ranging from 5\% to 8\%: the larger the pin is, the more important the mechanical constraint on it. Thus, configurations with large pins and thin cladding
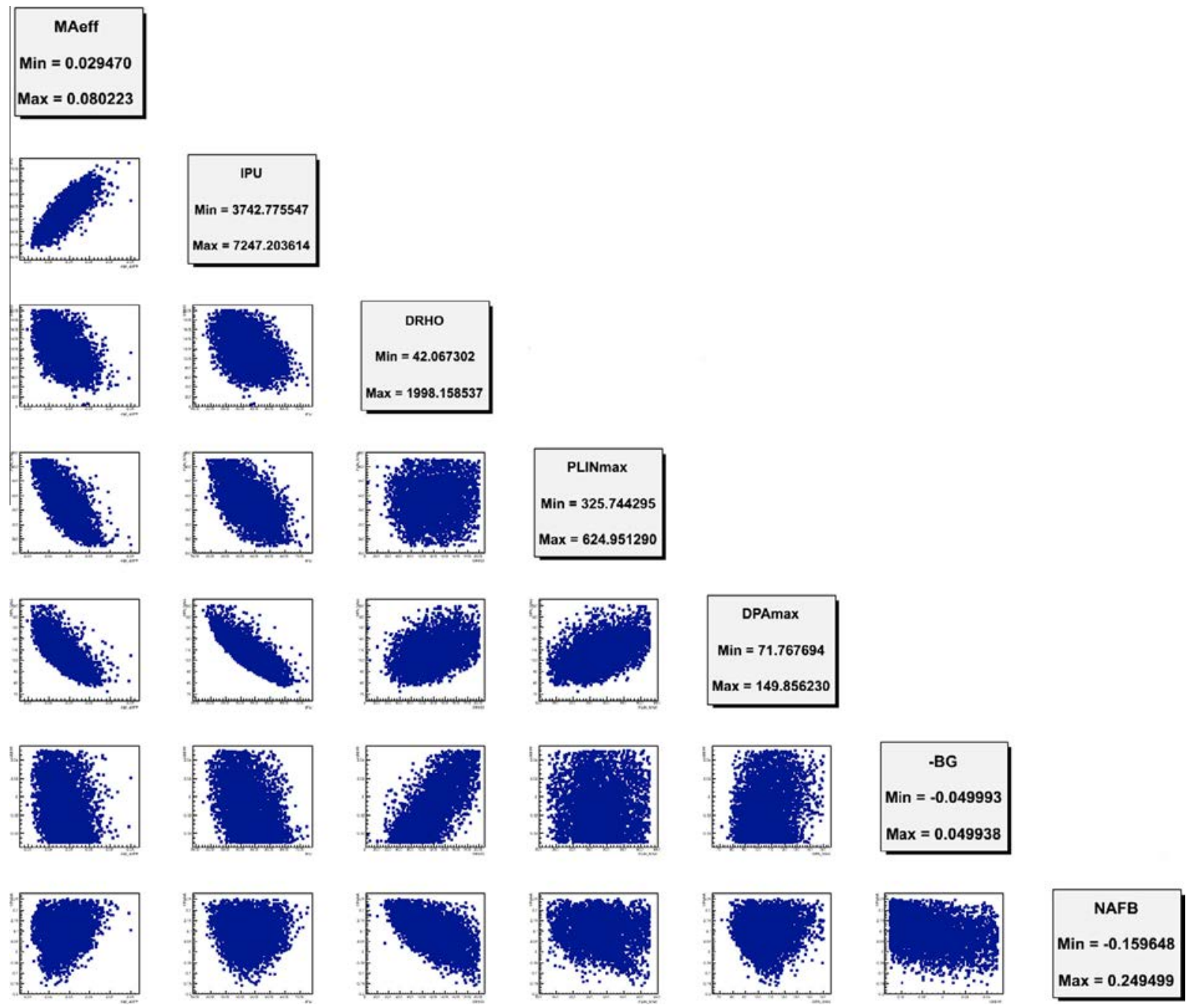

Fig. 9. Two dimensional projection of the Pareto front. 

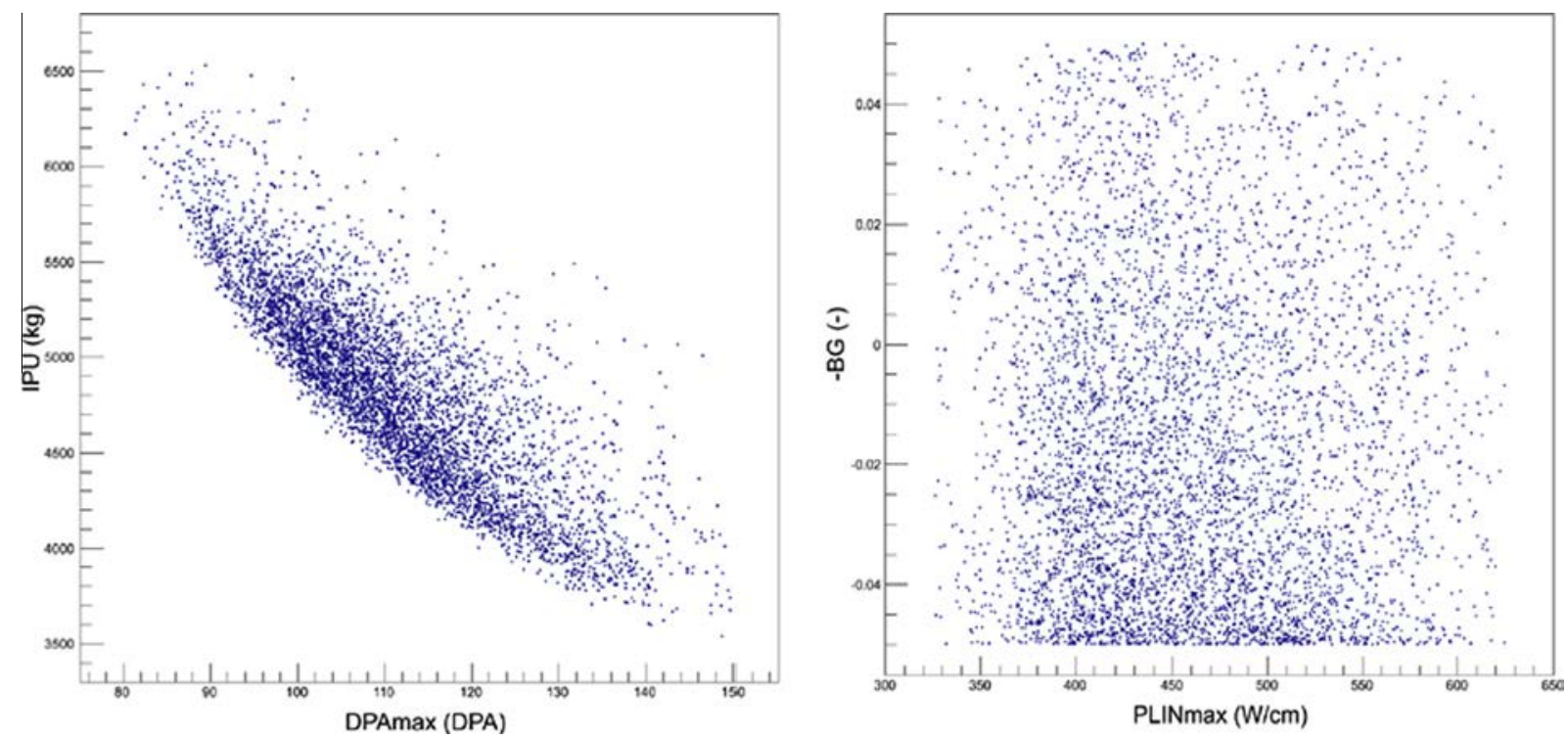

Fig. 10. Highly negatively correlated performances (left) and weakly correlated ones (right).

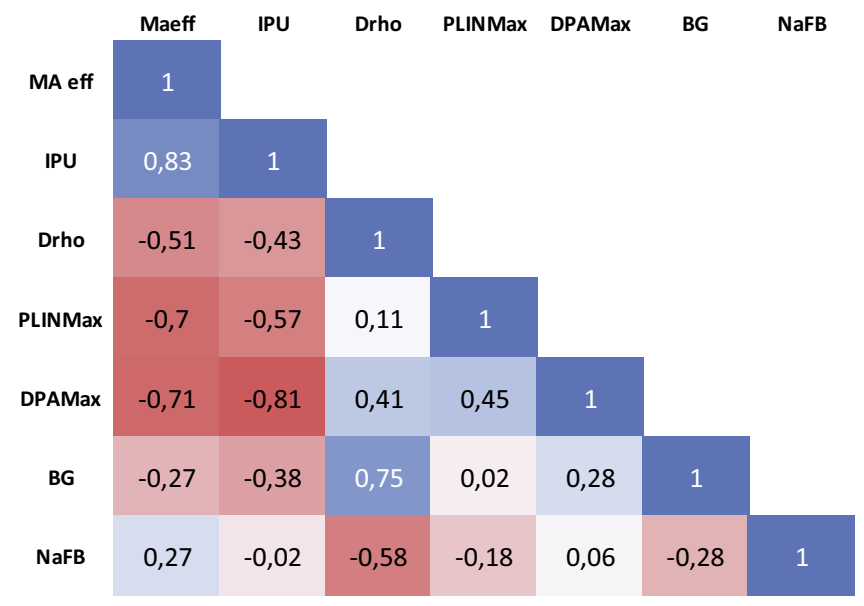

Fig. 11. Spearman's correlation matrix between performance variables.

should be avoided, to ensure good mechanical resistance of the later during irradiation (feedback from French operation of SFRs showed the viability of these boundaries).

\subsection{First optimization exercise}

The first Pareto front is found by selecting optimization variables retained for $\mathrm{CFV}$-v1 core, i.e.:

- only one enrichment ratio (internal/external cores), no axial enrichment,

- only one internal pellet radius,

- no minor actinides dilution in the fuel.

Thanks to the use of M-M, several thousand of configurations (5000) in the Pareto front can be quickly obtained by running an evolutionary algorithm. This process lasts less than an hour. Results in two dimensional projections are shown in Fig. 9.

The shape of this scatter plot is a first piece of information about how antagonist the performances can be. The unfilled areas correspond to the "physically impossible" combinations (Fig. 10).
Two selected zooms in Fig. 4 are reproduced in Fig. 5. The first one (left) reproduces the correlation between plutonium inventory (IPU) and maximal damage to the cladding (DPA $A_{\text {max }}$ ); one can observe that a low IPU is antagonist with a low DPA max. The blank zone in the lower left corner shows the absence of configuration exhibiting good score in both performances. At the opposite a spread scatter pattern (right) shows no antagonism between getting a good breeding gain (BG) and getting enhanced maximum linear power $\left(\mathrm{PLIN}_{\mathrm{max}}\right)$, or maximum dose on the cladding.

Fig. 6 shows the trade-off matrix computed on this Pareto front. The Spearman's correlation coefficients are computed. A positive coefficient means that ranks of the two performances are globally following the same trend. The improvement of one of the performance can go along with the improvement of the second. This is, notably, the case for minor actinides production and plutonium inventory, or breeding gain and reactivity swing.

A negative coefficient highlights antagonist performances, the antagonism being linked to the absolute value of the correlation coefficient. Unfortunately, some performances are strongly negatively correlated, such as maximal dose to the cladding vs. minor actinides effective production and plutonium inventory. The sodium feedback coefficient (last line) exhibits mainly negative or slightly positive correlation values, meaning that a dedicated improvement of this performance will deteriorate most of the others.

Distributions of optimization parameters also allow highlighting trends on the Pareto front. Some of them are depicted in Fig. 12. Optimal configurations are all found to have a number of fuel rings ranging from 8 to 11 and an external pellet radius ranging from $0.32 \mathrm{~cm}$ to $0.52 \mathrm{~cm}$ (top-left of Fig. 12). In other words, configurations outside these bounds cannot be Pareto optimal regarding the 7 defined performances.

Irradiation cycle duration distribution (top-right of Fig. 12) shows that most of the optimal configurations have a shorter cycle than the CFV-v1 reference core (for which the cycle lasts for 360 EFPD), the objective of CFV core to maximize this duration appears here as being hard to achieve.

Spacer wire diameter distribution (bottom-left of Fig. 12) shows the advantage on the neutronics point of view to design a compact fuel pin lattice (thin spacer wire). Enrichment ratio between inner and outer core (bottom-right of Fig. 12) confirms also the tendency 

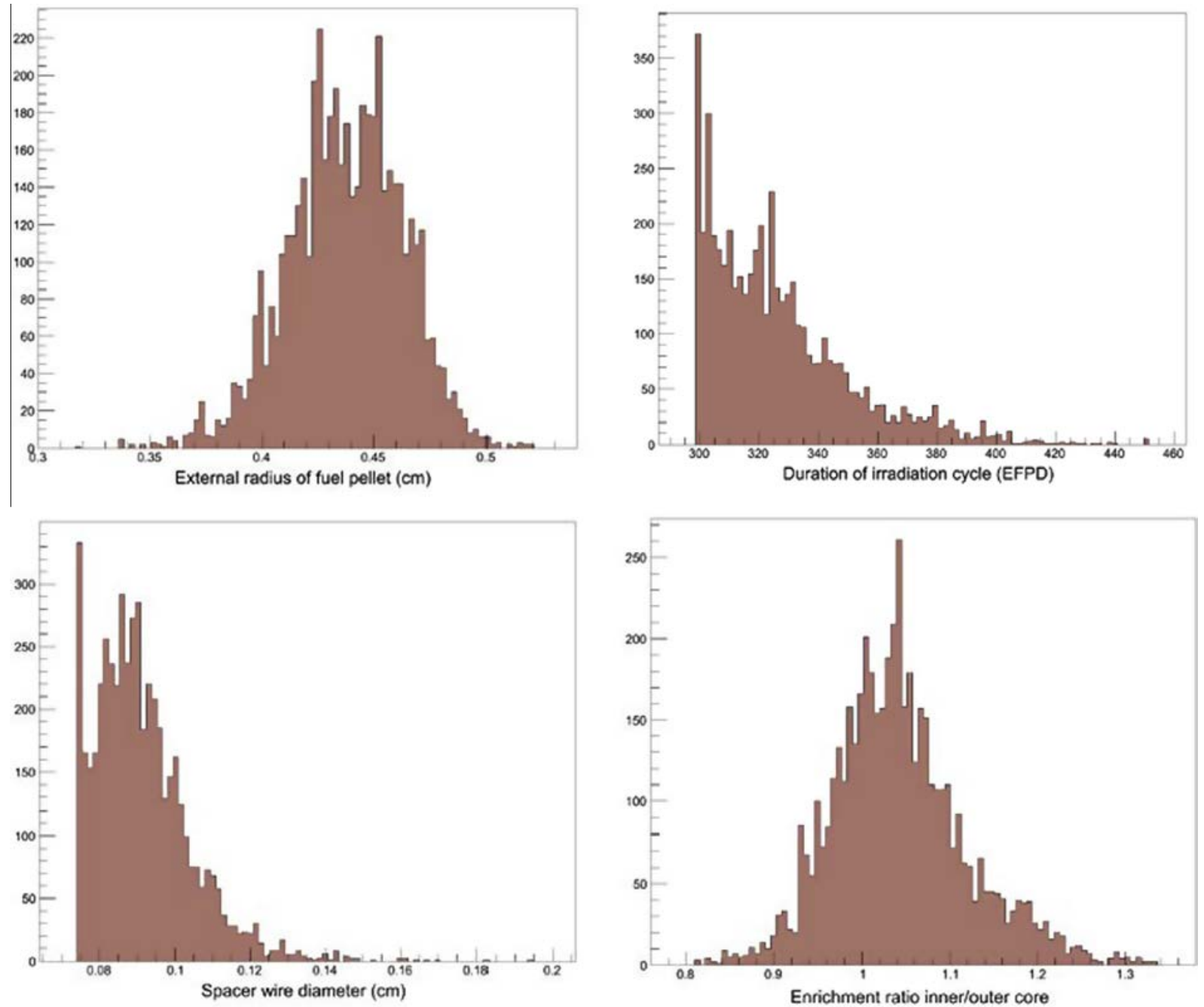

Fig. 12. Distribution of some optimization parameters in the Pareto set.

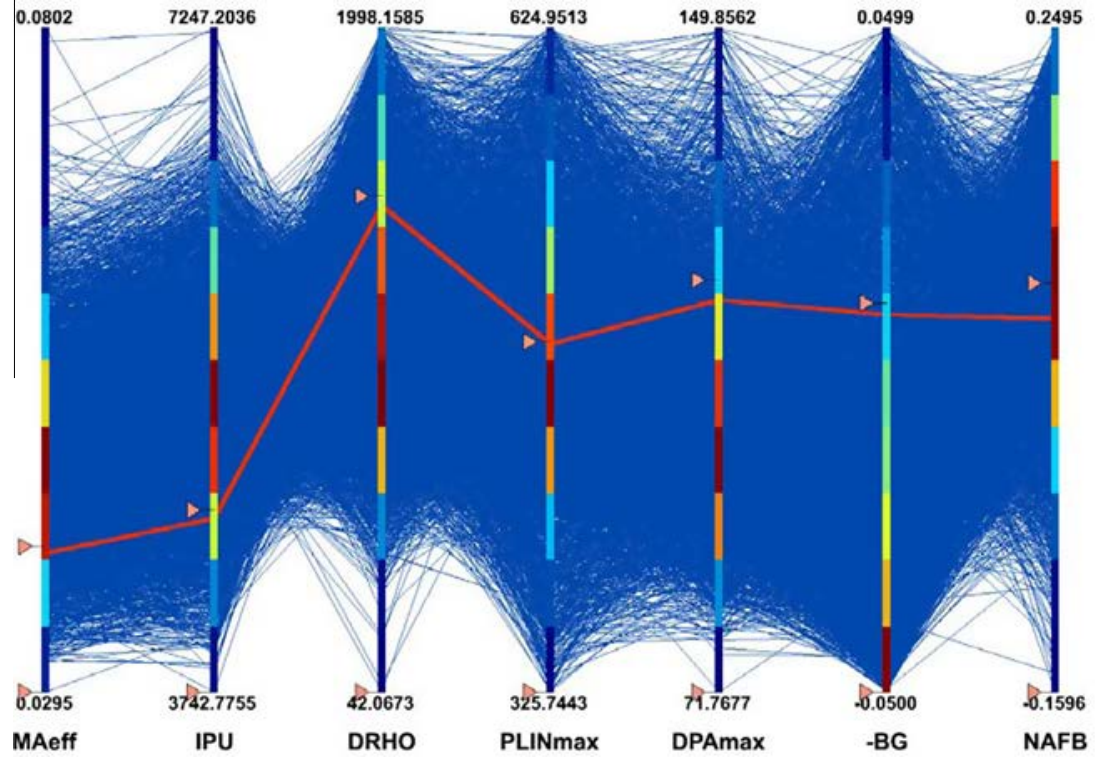

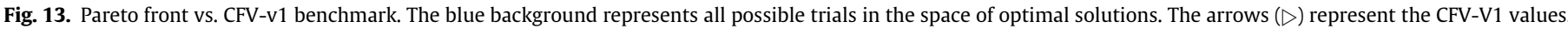
Only one solution is optimum. (For interpretation of the references to colour in this figure legend, the reader is referred to the web version of this article.) 

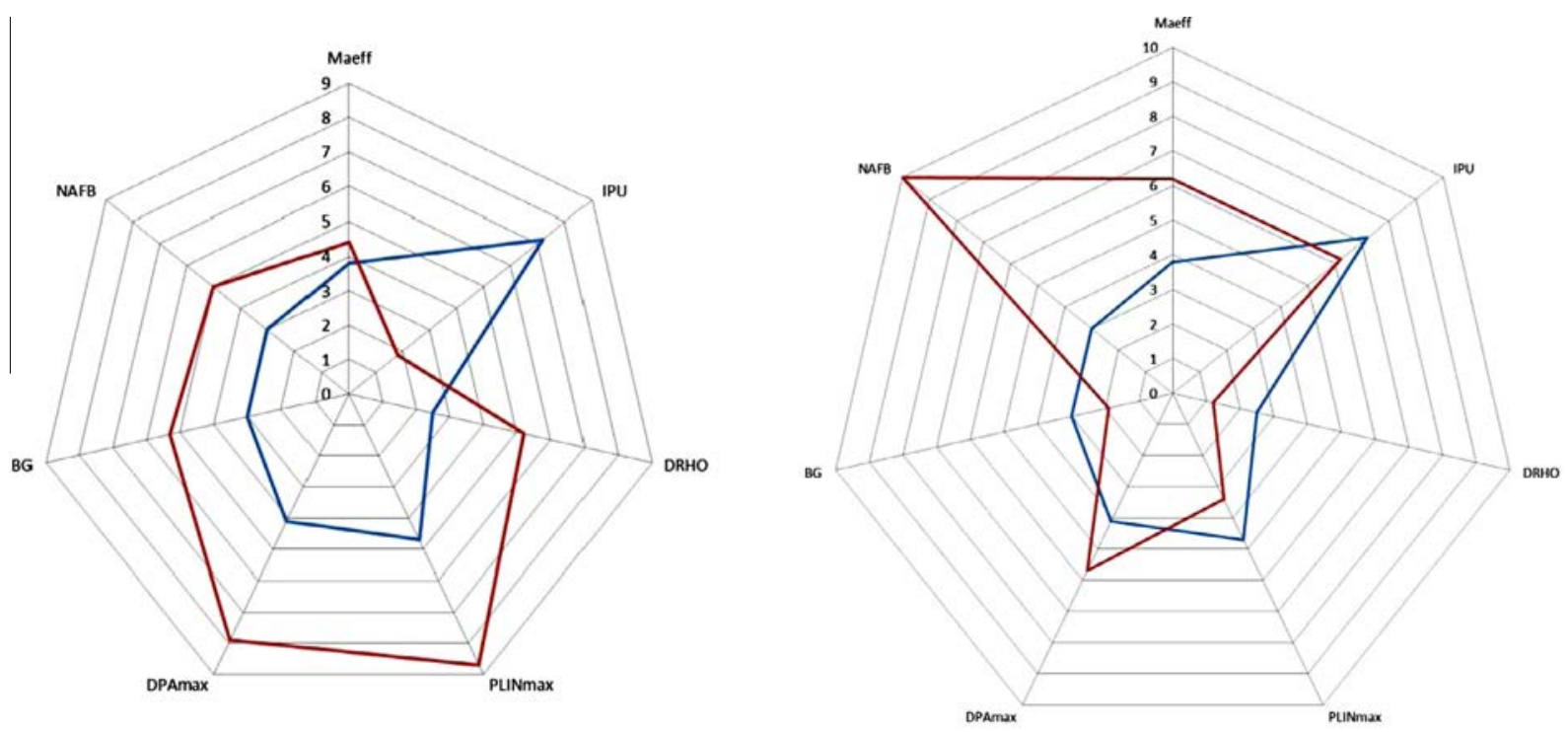

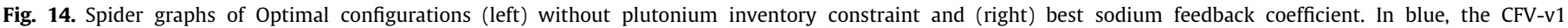
characteristics for comparison.

in the Pareto set to have a slightly over enriched inner core compared to the outer one.

The Pareto front is depicted in a parallel coordinate type diagram on Fig. 13 (reduced here for the sake of simplicity). Each of the vertical axes represents one of the 7 retained performances. The bottom of each axis corresponds to the best values for this specific optimization parameter. Then, one applies several constraints to each performance in order to select different core configuration profiles.

One first selects a set of constraints corresponding to the value of the CFV-v1 benchmark, plotted in red line on Fig. 13. As pointed out, no other configuration (no additional red line) is found by the metamodels, optimizing simultaneously all the performances of this benchmark.

This tends to prove that the CFV-v1 benchmark is Pareto optimal regarding the considered problem. With respect to all performances criteria, the CFV-v1, designed using Best Estimate deterministic schemes, is optimum on the neutronics point of view.

The obtained Pareto front also highlights that the benchmark configuration is on the upper side for some performances: maximal dose to the cladding, breeding gain, and reactivity swing. It appears also that the sodium thermal feedback coefficient (considered as one of the main objectives of the CFV design) can be further improved.

\subsection{Constraints ranking and relaxation}

Enabling some constraints to be relaxed from their bounds this is a decision makers' preference - can be an efficient technique to select relevant configurations among the several thousand ones from the Pareto set produced. As an example of the method's efficiency, two core configurations are highlighted in the followings:

- The first one is obtained by relaxing the Pu inventory at a lower value than the CFV-V1 reference core. The results are reproduced on Fig. 9-left.

- The second one is obtained by improving at best the sodium void feedback coefficient. The results are reproduced on Fig. 9-right.
To illustrate the improvement/degradation of each performance index, a grade $G_{i}$ between 0 and 10 is given for each performance $y_{i}$ regarding its position on the Pareto front. A grade of $10 y_{\max }$ (resp. 0 $y_{\min }$ ) corresponds to the highest (resp. lowest) grade and means that the configuration is the best (resp. the worst) found in the optimization process. The $G_{i}$ grades are calculated as follows:

$G_{i}=10 \frac{y_{\max }-y_{i}}{y_{\max }-y_{\min }}$ if $y$ should be minimized

$G_{i}=10 \frac{y_{i}-\min }{y_{\max }-y_{\min }}$ if $y$ should be maximized

Fig. 14 (left) shows the obtained results if the constraint of having a plutonium inventory lower than the CFV-v1 one is discarded.

As pointed out, the configuration, improves all performances but the plutonium content one (grade goes from 7 to 2 ), compared to CFV-v1:

- $\mathrm{DPA}_{\max }$ grade goes from 4 to 8 .

- BG grade goes from 3 to 5.

- PLIN $_{\max }$ grade goes from 5 to 8.

- NAFB and DRHO grades are improved from 3 to 5.

The second configuration is selected to reach the best sodium feedback coefficient. The results are shown in the right section of Fig. 9. As exhibited by the figure, maximizing the sodium feedback performance induces a significant improvement of the waste production (from 4 to 6 ) and slightly increase the maximal allowed dose to the cladding (from 4 to 5 ) as expected from the Spearman correlations results of Fig. 6 (both coefficients are positive). All the others performances are slightly lowered.

\section{Additional optimization studies}

\subsection{Additional degrees of freedom}

Another strength of the methodology is the possibility to explore effects of innovative parameter designs. The study is extended to include 10 additional degrees of freedom that are: 


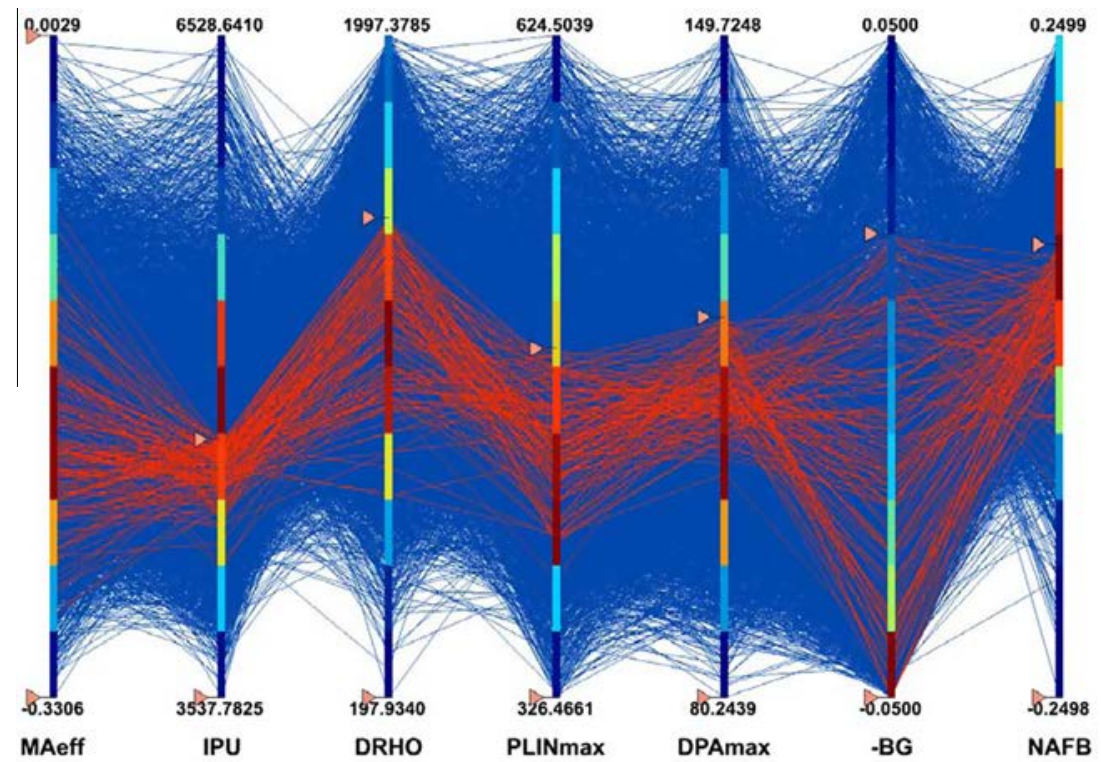

Fig. 15. Innovative concepts optimal configurations. All red configurations issued from the optimization behave better than the target CFV-V1 configuration.

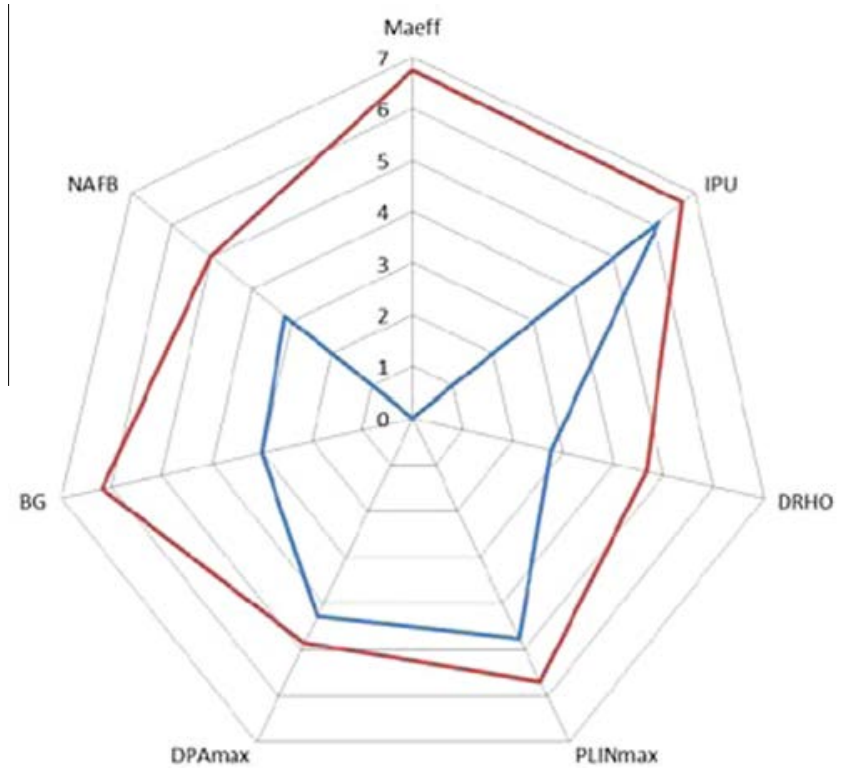

Fig. 16. Spider graph of Optimal configuration with positive breeding gain and negative sodium feedback.

- 5 enrichment zones (3 fissile, 2 fertile); compared to 2 previously.

- 5 internal pellet diameters (3 fissile, 2 fertile); compared to 1 initially.

- 2 minor actinides dilution rates in the fuel (homogeneous dilution in fertile and fissile zones); as none was considered in the previous section.

The Multi-Objective Optimization algorithm is run again with the same set of constraints as previously. Five thousand new optimal configurations are then obtained in the new Pareto front. Among these, only configurations improving all the performances at once are selected. Results are shown in Fig. 15. As expected, and because of the higher number of degrees of freedom, several configurations are found to be more optimized than the CFV-v1 benchmark, identified by the arrows $(\triangleright)$ on each axis. In this frame-

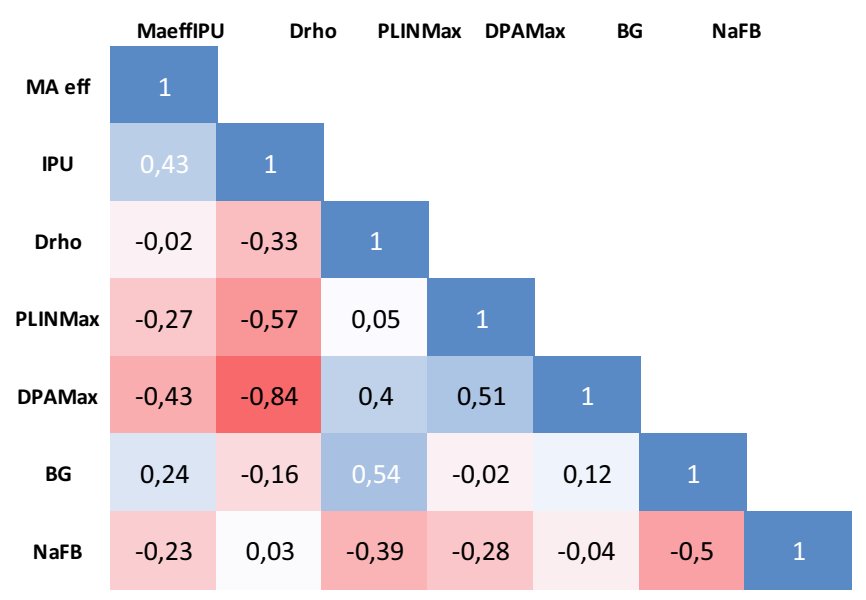

Fig. 17. Correlation matrix for the innovative concepts.

work, including more degrees of freedom, CFV-v1 is then shown to be not optimal anymore, as expected. Except for the sodium feedback coefficient and the plutonium inventory for which the gain are relatively small, all the other performances can be greatly improved with no significant degradation on any other side (Fig. 16).

Some boundaries of the Pareto front are also improved, especially for the minor actinide production for which both upper and lower boundaries are lowered. The minimum attainable for plutonium inventory (from $3750 \mathrm{~kg}$ to $3550 \mathrm{~kg}$ ), sodium feedback (from -0.16 to $-0.25 \mathrm{pcm} /{ }^{\circ} \mathrm{C}$ ) are also improved. As in the previous optimization study all but two of the optimization parameters vary within their whole range. For all optimal configurations, external radius of fuel pellet is ranging from $0.34 \mathrm{~cm}$ to $0.50 \mathrm{~cm}$ and number of fuel rings is ranging from 8 to 12 . Distributions of the additional optimization parameters (not shown here) lead to the following observations:

- Dilutions of minor actinides in both fissile and fertile zones are equally spread in the Pareto set. They are also not correlated with each other. 


\section{Plutonium Inventory $(\mathrm{kg})$}

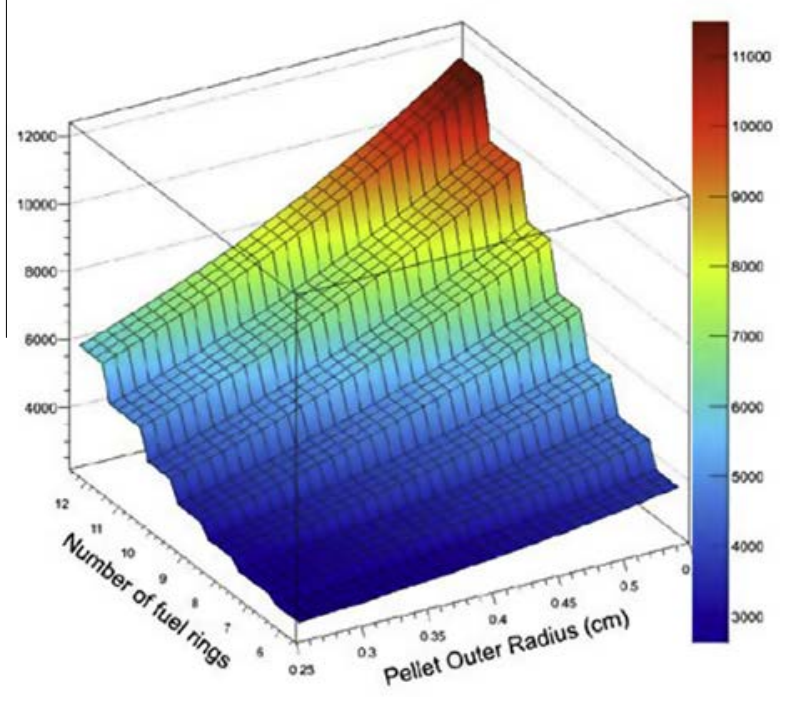

Plutonium Inventory $(\mathrm{kg})$

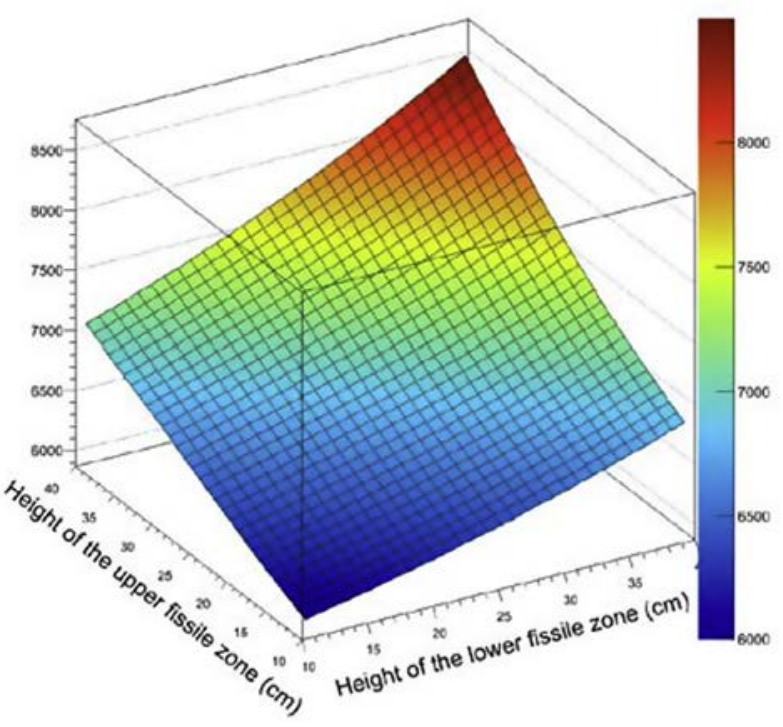

Fig. 18. Three dimensional plots of plutonium inventory metamodel.
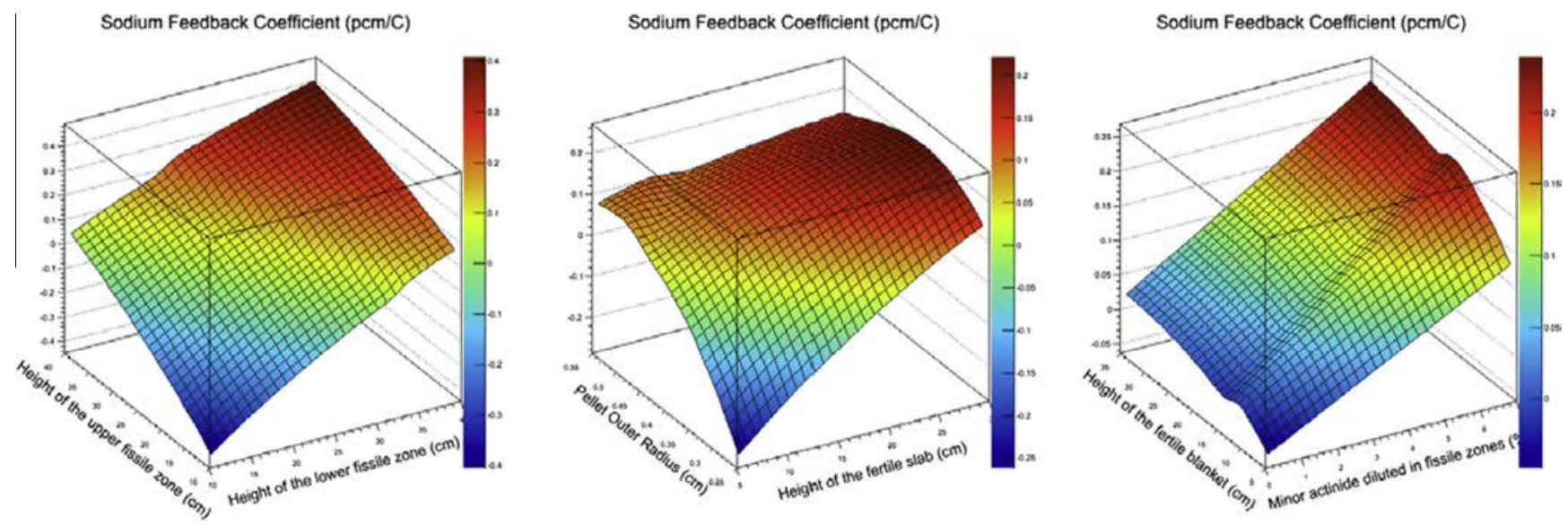

Fig. 19. Three dimensional plots of sodium feedback coefficient metamodel.

Table 10

Validation results on reduced Pareto set.

\begin{tabular}{|c|c|c|c|c|}
\hline Performances & Units & Maximum discrepancy & Error of simplified scheme & Reference scheme uncertainties \\
\hline Plutonium Inventory & $\mathrm{kg}$ & 65 & 68 & $\mathrm{n} / \mathrm{a}$ \\
\hline Breeding gain & - & 0.002 & 0.0 & 0.05 \\
\hline Reactivity swing & $\mathrm{pcm}$ & 114 & 10 & $>300$ \\
\hline Maximal Linear Power & $\mathrm{W} / \mathrm{cm}$ & 10 & 48 & $>30$ \\
\hline Dose to the cladding & DPA & 2.0 & 2.0 & 15 \\
\hline Minor actinide Production & $\mathrm{kg} / \mathrm{EFPD}$ & 0.002 & 0.002 & 0.007 \\
\hline Sodium feedback & $\mathrm{pcm} /{ }^{\circ} \mathrm{C}$ & 0.01 & 0.018 & 0.02 \\
\hline
\end{tabular}

- Enrichment ratios distributions confirm the tendency of having an inner core more enriched than the outer one. However, the additional parameters highlight the trend to over enrich the upper fissile zone compared to the lower one. The clear advantage of introducing axial heterogeneities in the enrichment in thus shown here.

- No clear trends or correlation have been identified for the different internal pellet radii.

Among these configurations, it is then easy to select the one fulfilling the project requirements in the best possible way. The spider graph on Fig. 11 reproduces a Pareto optimal configuration selected for its global negative sodium feedback and its positive breeding gain, along with a homogeneous improvement of all other performances.

The associated Spearman correlation matrix has also been computed, and is reproduced on Fig. 17. It allows to directly measure the impact of innovative configurations on the trade-off. As expected, values associated with waste production are the most noticeably modified. This performance becomes more antagonists with the plutonium inventory and sodium feedback but becomes more compatible with the reactivity swing, breeding gain, maximal linear power and cladding damage. 
Table 11

Sobol indices for Plutonium Inventory performance.

\begin{tabular}{|c|c|c|c|}
\hline Variable & First order Sobol indices (\%) & Total Order Sobol indices (\%) & Trend of variation for improvement \\
\hline Number of fuel rings & 66 & 72 & Decrease \\
\hline Pellet external diameter & 21 & 25 & Decrease \\
\hline Fertile slab Height & 1.6 & 2.0 & Decrease \\
\hline Lower fissile height & 1.5 & 2.0 & Decrease \\
\hline Upper fissile height & 1.3 & 1.5 & Decrease \\
\hline Spacer wire thickness & 1.3 & 1.3 & Decrease \\
\hline
\end{tabular}

Table 12

Sobol indices for sodium feedback coefficient.

\begin{tabular}{|c|c|c|c|}
\hline Variable & $\begin{array}{l}\text { First order } \\
\text { Sobol Indices } \\
(\%)\end{array}$ & $\begin{array}{l}\text { Total order } \\
\text { Sobol indices } \\
(\%)\end{array}$ & $\begin{array}{l}\text { Trend of variation } \\
\text { for improvement }\end{array}$ \\
\hline Lower fissile height & 31 & 34 & Decrease \\
\hline Upper fissile height & 23 & 27 & Decrease \\
\hline Fertile Slab height & 8.8 & 12 & Decrease \\
\hline Outer pellet radius & 7.2 & 11 & Decrease \\
\hline $\begin{array}{l}\text { Minor actinide } \\
\text { content in the } \\
\text { fissile zones }\end{array}$ & 4.6 & 5.2 & Decrease \\
\hline Fertile blanket height & 3.9 & 5.4 & Decrease \\
\hline
\end{tabular}

\subsection{Validation of optimization results}

The last step of the methodology consists in validating the optimization results. Two hundred configurations have been taken from the last Pareto front and recalculated using the optimized neutron physics calculation scheme instead of the metamodels. The correlation values of both initial Pareto set and its subpopulation are very close (the maximum differences do not exceed the percent). The sub-population has been considered as statistically representative. The maximum errors found for each performance are shown in the Table 10.

Except for the reactivity loss per cycle, all the maximum errors on the optimal configurations are lower than the initial error produced by the simplified calculation scheme. Concerning the reactivity swing, attention should be paid to the error bounds. Two configurations with reactivity swing difference lower than $100 \mathrm{pcm}$ have to be further investigated in order to extract the best one as far as this criterion is concerned. However, error produced by this metamodel still remains below the reference scheme uncertainty. All the results are thus considered as satisfactory.

\section{Conclusions}

A full methodology for optimizing heterogeneous SFR core design from a neutronics point of view has been implemented and successfully applied. It allowed identifying an important set of potentially optimal configurations within a very large design space. The parameterization included up to thirty variables, with wide ranges. In such conditions, a classical parametric study using reference core codes and associated calculation schemes is almost impossible. In the method presented in this paper, all inputs were varied simultaneously, enabling a full exploratory, early design phase compatible methodology (Table 11).

A neutronics model, based on the ERANOS reference deterministic code for SFR studies at CEA has been built and validated on the available benchmark. Some assumptions have been made in order to adapt the reference Best Estimate calculation scheme to the optimization process, notably the decision to design the core regarding to its equilibrium state. Errors on benchmark have been found to fall within most of the reference uncertainties boundaries. Only local values of the maximal linear power are biased by a more important error. The absence of modeling of the fuel shuffling is considered as the main source of this deviation.

Based on this simplified deterministic neutron core calculation models, Artificial Neural Networks have been implemented as substitute models for all of the eleven performances of the core retained here. These performances have been defined in accordance with the main objectives of Generation IV reactors: safety, operability, ultimate wastes transmutation, and a mastered investment cost. A preliminary validation of the corresponding metamodels was performed before a global optimization study.

To cope with the intrinsic antagonism between all the performances estimators, metamodels have been used to perform Multi-Objective Optimizations. Large sets of optimal configurations (several thousands of individuals) have been identified thanks to an evolutionary algorithm. The optimality of the CFVv1 benchmark, considered here as the reference solution, has been confirmed. Progressive constraints relaxation allowed the identification of other potentially interesting configurations. As an example among others, the increase of initial plutonium core content can lead to improvements of considered safety estimators as well as for the breeding gain.

Statistical information has been produced from this whole set of optimal solutions to get correlations matrices between the considered performances, these correlations expressing their global trade-off. An insight has been given on their mutual antagonisms. Plutonium inventory has been shown to be antagonist with most of the other performance estimators. Sodium feedback coefficient also appears to be antagonist with most other performances. In particular, negative correlations have been found with other safety estimators such as maximum linear power and reactivity swing. The effects of core radial size have been highlighted: the obtained conclusions regarding height/diameter (H/D) ratio are similar to Sun's work (2012).

This optimization method and the same metamodels were also used to quantify the potentialities of design options such as increasing axial heterogeneities or diluting minor actinides in the fuel. New configurations, improving the CFV-v1 in all its initial performances have been found thanks to these additional degrees of freedom. Finally, a validation process has been applied on an optimal subset of configurations to ensure the qualities of the results. All of the maximum error bounds were found within the model uncertainties, except the reactivity swing which exhibits greater biases, roughly a hundred pcm. However this error has been considered as acceptable in the early design phase.

Future work will concentrate on developing a multi-physics calculation chain based on metamodels. Multi-criteria optimization will be carried out on some thermal-hydraulics transients. The results will be presented in two forthcoming papers.

\section{Acknowledgements}

The authors express their sincere and grateful appreciation to their colleagues of DER/SPRC/LEDC from the French Atomic Energy and Alternative Energies Commission who provided thoughtful reviews and useful suggestions for this work. 


\section{Appendix A. Some details of the Global Sensitivity Analysis}

This appendix details the Global Sensitivity Analysis performed in Section 6. For Plutonium inventory, the performances described in Section 4 are detailed in Table 2, with the values of both first and total indices for influent variables (first index above one percent). This information is completed by 3D plots (cf. Fig. 18) to show the trends of the responses functions. In these graphs, two of the influent parameters are modified within their whole range while the remaining 28 parameters are kept constant to their reference value (cf. Tables 4 and 5). The same results are shown in Table 12 and Fig. 19 for the sodium dilatation feedback coefficient.

\section{Appendix B. List of acronyms}

\begin{tabular}{|c|c|}
\hline ANN & Artificial Neural Networks \\
\hline $\mathrm{BE}$ & Best Estimate \\
\hline BEMUSE & $\begin{array}{l}\text { Best Estimate Methods in Uncertainty and } \\
\text { Sensitivity Evaluation }\end{array}$ \\
\hline BG & Breeding Gain \\
\hline $\mathrm{BOC}$ & Beginning Of Cycle \\
\hline BOL & Beginning Of Life \\
\hline CEA & $\begin{array}{l}\text { Commissariat à l'Energie Atomique et aux Energies } \\
\text { Alternatives (French Atomic Energy and } \\
\text { Alternative Energies Commission) }\end{array}$ \\
\hline CCD & Central Composite Design \\
\hline CFV & Coeur Faible Vidange (Low Void effect Core) \\
\hline CSNI & Committee on the Safety of Nuclear Installations \\
\hline DOD & D-Optimal Design \\
\hline DoE & Department of Energy \\
\hline DOE & Design of Experiment \\
\hline DPAmax & $\begin{array}{l}\text { Maximal dose to the cladding during fuel assembly } \\
\text { lifetime }\end{array}$ \\
\hline DRHO & Reactivity loss per cycle \\
\hline EFPD & Equivalent Full Power Day \\
\hline EFR & European Fast Reactor \\
\hline EOC & End Of Cycle \\
\hline EOL & End Of Life \\
\hline ERANOS & European Reactor Analysis Optimized code System \\
\hline FARM & FAst Reactor Methodology \\
\hline GA & Genetic Algorithm \\
\hline GIF & Generation IV International Forum \\
\hline GSA & Global Sensitivity Analysis \\
\hline IPU & Plutonium Inventory \\
\hline IRSN & $\begin{array}{l}\text { Institut de Radioprotection et Sûreté Nucléaire } \\
\text { (Radioprotection and Nuclear Safety Institute) }\end{array}$ \\
\hline LHD & Latin Hypercube Design \\
\hline LOCA & Loss of Coolant Accident \\
\hline LOFT & Loss of Flow Transient \\
\hline LT & LifeTime \\
\hline MAeff & Minor Actinide effective production \\
\hline METRO & $\begin{array}{l}\text { Mathematical Estimation of Transients for Reactor } \\
\text { design Orientation }\end{array}$ \\
\hline MM & Meta Models \\
\hline MOO & Multi-Objective Optimization \\
\hline NAFB & Sodium FeedBack coefficient \\
\hline OAD & Orthogonal Array Design \\
\hline PCT & Peak Cladding Temperature \\
\hline PLINmax & Maximal Linear power \\
\hline $\mathrm{RBF}$ & Radial Basis Function \\
\hline RDO & Robust Design Optimization \\
\hline SDDS & SHADOC-based Design Development System \\
\hline
\end{tabular}

UCRW Unprotected Control Rod Withdrawal

UD

ULOF Uniform Design

ULOHS Unprotected Loss of Heat Sink

ULOSSP Unprotected Loss of Station Supply Power

WGAMA Working Group on Accident Management and Analysis

\section{References}

Barjot, F., Schmitt, D., Venard, C., 2014. Multi-physics and multi-objective optimization methodology for sodium cooled fast reactor conception. Proceedings of ICAPP 2014, Paper 14176, Charlotte, USA, April 6-9.

Buiron, L., Fontaine, B., Andriolo, L., 2012. Transmutation abilities of the SFR low void effect core concept CFV 3600 MWth. Proceedings of ICAPP 2012, Chicago, USA, June 24-28, Paper 12029.

Chenaud, M-S et al., 2013. Status of the ASTRID core at the end of the preconceptual design phase 1. Proceedings of ICAPP 2013, Jeju Island, Korea, April 14-18, Paper FA169.

Forrester, A.I.J., Sóbester, A., Keane, A.J., 2008. Engineering Design via Surrogate Modeling: A Practical Guide. John Wiley \& Sons Ltd., ISBN 978-0-470-06068-1.

Fouet, F. et al., 2007. Sensitivity analysis by the use of a surrogate model in LBLOCA: LOFT L2-5 with CATHARE-2 V2.5 Code. Proc. Int. Conf NURETH-12, Pittsburgh.

Gaudier, F., 2010. URANIE: the CEA/DEN uncertainty and sensitivity platform. Sixth International Conference on Sensitivity Analysis of Model Output, Procedia Social and Behavioral Sciences 2, 7660-7661.

GIF, 2012. Symposium Proceedings, Annual Report, San Diego, USA (CA).

Giunta, A. A., Wojtkiewicz Jr, S. F., and Eldred, M. S., 2001. Overview of Modern Design of Experiments Methods for Computational Simulations. AIAA paper 2003-649.

Hourcade, E., Jasserand, F., Ammar, K., 2013. SFR core design: a system driven multicriteria optimization exercise with TRIAD, Fast Reactor Conference FR13, IAEA. Paper 421.

Ingremeau, J-J., 2011. Méthodologie d'optimisation d'un cœur de réacteur à neutrons rapides, application à l'identification de solutions permettant des performances accrues ( $\mathrm{PhD}$ thesis). Université Paris Sud XI.

Iooss, B. et al., 2010. Numerical studies of the metamodel fitting and validation processes. Int. J. Adv. Syst. Measur. 3, 11-21.

Jin, R., Chen, W., Simpson, T., 2001. Comparative studies of metamodeling techniques under multiple modeling criteria. J. Struct. Multi. Optim. 23 (1).

Kirkpatrick, S., Gelatt, C.D., Vecchi, M.P., 1983. Optimization by simulated annealing. Science 220 (4598), 671-680.

Lin, Y., 2004. An Efficient Robust Concept Exploration Method and Sequential Exploratory Experimental Design (Ph.D. thesis). Georgia Institute of Technology.

Perez, M. et al., 2010. Main Results of Phase IV BEMUSE Project: Simulation of LBLOCA in a NPP Vol. 2010. Science and Technology of Nuclear Installations.

Reid, S, 2014. <http://www.turingfinance.com/misconceptions-about-neuralnetworks/>.

ROOT, 1998. ROOT: an object-oriented data analysis framework. Linux J. 51.

Ruggieri, J.M., Tommasi, J., Lebrat, J.F, Suteau, C. Plisson-Rieunier, D., De Saint Jean, C., Rimpault, G., Sublet, J-C., 2006. ERANOS2.1: the international code system for Gen-IV fast reactor analysis. Proc. Int. Conf. ICAPP'06, Reno, USA.

Saltelli, A., Annoni, P., Azzini, I., Campolongo, F., Ratto, M., Tarantola, S., 2010. Variance based sensitivity analysis of model output. Design and estimator for the total sensitivity index. Comput. Phys. Commun. 181, 259-270.

Ruggieri, J.M., Tommasi, J., Lebrat, J.F, Suteau, C., Plisson-Rieunier, D., De Saint Jean, C. Rimpault, G., Sciora, P., et al., 2011. Low Void Effect Core Design Applied on 2400 MWth SFR Reactor. International Congress on Advances in Nuclear Power Plants - ICAPP'11, Nice, France, May 2-6.

Simpson, T.W., Peplinski, J., Koch, P.N., Allen, J.K., 1997. On the Use of Statistics in Design and the Implications for Deterministic Computer Experiments. Design Theory and Methodology - DTM'97, Sacramento, CA, ASME, DETC97/DTM-3881.

Stauff, N., Buiron, L., Fontaine, B., Rimpault, G., 2013. Methodology for designing a sodium-cooled fast reactor with inherent safety. Nucl. Technol. 181.

Sun, K., 2012. Analysis of Advanced Sodium-cooled Fast Reactor Core Designs with Improved Safety Characteristics (Ph.D. thesis). Ecole Polytechnique de Lausanne.

Turinsky, P., 2012. Advances in multi-physics and high performance computing in support of nuclear reactor power systems modeling and simulation. Nucl. Eng. Technol. 44 (2).

Waltar, A.E., Reynolds, A.B., 1981. Fast Breeder Reactors. Pergamon Press.

Wang, G.G., Shan, S., 2005. Review of metamodeling techniques for product design with computation-intensive processes. Proc. Can. Eng. Edu. Assoc.

Yang, W.-S., 2012. Fast reactor physics and computational methods. Nucl. Energy Eng. 44 (2). 